CRYSTALLOGRAPHIC COMMUNICATIONS

ISSN 2056-9890

Received 12 June 2019

Accepted 21 June 2019

Edited by C. Rizzoli, Universita degli Studi di Parma, Italy

Keywords: crystal structure; acetohydrazides; thiophene; Hirshfeld analysis.

CCDC references: 1935593; 1935592

Supporting information: this article has supporting information at journals.iucr.org/e

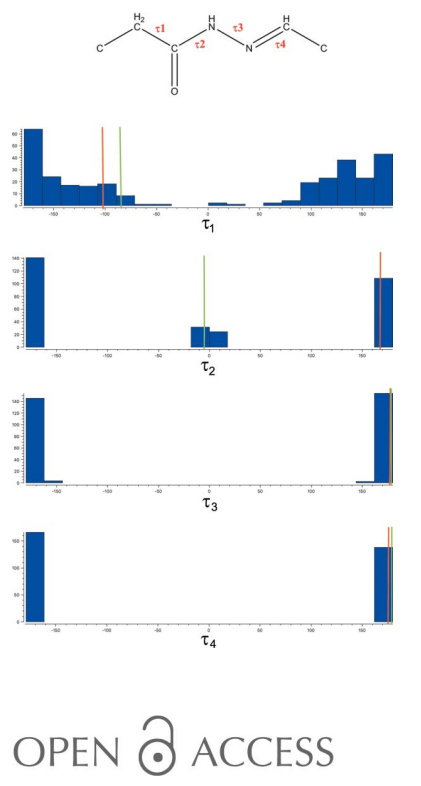

\section{Crystal structure of two $N^{\prime}$-(1-phenylbenzylidene)- 2-(thiophen-3-yl)acetohydrazides}

\author{
Trung Vu Quoc, ${ }^{\mathrm{a} *}$ Linh Nguyen Ngoc, ${ }^{\mathrm{a}}$ Duong Tran Thi Thuy, ${ }^{\mathrm{a}, \mathrm{b}}$ Manh Vu Quoc, ${ }^{\mathrm{c}}$ \\ Thien Vuong Nguyen, ${ }^{\mathrm{d}, \mathrm{e}}$ Yen Oanh Doan Thi ${ }^{\mathrm{f}}$ and Luc Van Meervelt ${ }^{\mathrm{g} *}$
}

\begin{abstract}
${ }^{\mathbf{a}}$ Faculty of Chemistry, Hanoi National University of Education, 136 Xuan Thuy, Cau Giay, Hanoi, Vietnam, ${ }^{\mathbf{b}}$ Bien Hoa Gifted High School, 86 Chu Van An Street, Phu Ly City, Ha Nam Province, Vietnam, ${ }^{\mathrm{C}}$ Faculty of Foundation Science, College of Printing Industry, Phuc Dien, Bac Tu Liem, Hanoi, Vietnam, IInstitute for Tropical Technology, Vietnam Academy of Science and Technology, 18 Hoang Quoc Viet, Cau Giay, Hanoi, Vietnam, ${ }^{\mathbf{e}}$ Graduate University of Science and Technology, Vietnam Academy of Science and Technology, 18 Hoang Quoc Viet, Cau Giay, Hanoi, Vietnam, 'Publishing House for Science and Technology, Vietnam Academy of Science and Technology, 18 Hoang Quoc Viet, Cau Giay, Hanoi, Vietnam, and ${ }^{\mathbf{g}}$ Department of Chemistry, KU Leuven, Biomolecular Architecture, Celestijnenlaan 200F, Leuven (Heverlee), B-3001, Belgium. *Correspondence e-mail: trungvq@hnue.edu.vn, Luc.VanMeervelt@kuleuven.be
\end{abstract}

The synthesis, spectroscopic data, crystal and molecular structures of two $N^{\prime}$-(1phenylbenzylidene)-2-(thiophen-3-yl)acetohydrazides, namely $N^{\prime}$-[1-(4-hydroxyphenyl)benzylidene]-2-(thiophen-3-yl)acetohydrazide, $\quad \mathrm{C}_{13} \mathrm{H}_{10} \mathrm{~N}_{2} \mathrm{O}_{2} \mathrm{~S}$, (3a), and $N^{\prime}$-[1-(4-methoxyphenyl)benzylidene]-2-(thiophen-3-yl)acetohydrazide, $\mathrm{C}_{14} \mathrm{H}_{14} \mathrm{~N}_{2} \mathrm{O}_{2} \mathrm{~S}$, (3b), are described. Both compounds differ in the substituent at the para position of the phenyl ring: $-\mathrm{OH}$ for $(3 \mathrm{a})$ and $-\mathrm{OCH}_{3}$ for (3b). In (3a), the thiophene ring is disordered over two orientations with occupancies of 0.762 (3) and $0.238(3)$. The configuration about the $\mathrm{C}=\mathrm{N}$ bond is $E$. The thiophene and phenyl rings are inclined by $84.0(3)$ and $87.0(9)^{\circ}$ for the major- and minor-occupancy disorder components in (3a), and by $85.89(12)^{\circ}$ in (3b). Although these dihedral angles are similar, the conformation of the linker between the two rings is different [the $\mathrm{C}-\mathrm{C}-\mathrm{C}-\mathrm{N}$ torsion angle is $-a c$ for (3a) and $-s c$ for ( $3 b$ ), while the $\mathrm{C} 6-\mathrm{C} 7-\mathrm{N} 9-\mathrm{N} 10$ torsion angle is $+a p$ for (3a) and $-s p$ for (3b)]. A common feature in the crystal packing of (3a) and (3b) is the presence of $\mathrm{N}-\mathrm{H} \cdots \mathrm{O}$ hydrogen bonds, resulting in the formation of chains of molecules running along the $b$-axis direction in the case of (3a), or inversion dimers for (3b). The most prominent contributions to the surface contacts are those in which $\mathrm{H}$ atoms are involved, as confirmed by an analysis of the Hirshfeld surface.

\section{Chemical context}

Acetohydrazides are considered to be good candidates for different pharmaceutical applications, including their use as antibacterial, antifugal, antimicrobial and anticonvulsant agents (Yadav et al., 2015; Bharti et al., 2010; Loncle et al., 2004; Papakonstantinou-Garoufalias et al., 2002). Moreover, many of them have shown analgesic and antiplatelet properties (Wardakhan et al., 2013). Combinations of acetohydrazide with other heterocyclic rings have also been investigated, such as the hydrazide-based 2-oxonicotinonitrile derivatives that are considered to be potential antimicrobial agents (El-Sayed et al., 2018).

As a continuation of our research (Nguyen et al., 2016; Vu et al., 2016, 2017) on the chemical and physical properties of novel polythiophenes, a new thiophene monomer-containing acetohydrazide has been prepared. We have synthesized two $N^{\prime}$-(1-(phenylbenzylidene)-2-(thiophen-3-yl)acetohydrazides and present here the spectroscopic data and crystal structures 
of the title compounds, together with the Hirshfeld surface analysis.

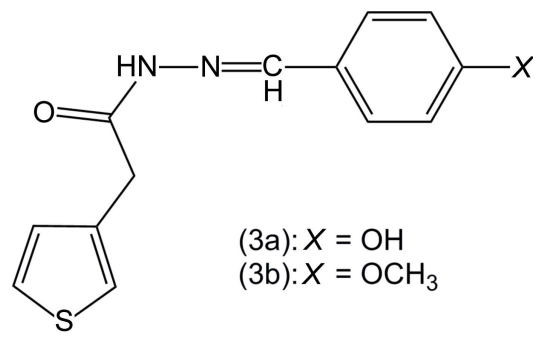

\section{Structural commentary}

The hydroxy derivative (3a) crystallizes in the orthorhombic space group Pbca. The thiophene ring is disordered over two sites (the major and minor components are labelled with the suffixes $A$ and $B$, respectively), corresponding to a rotation about the $\mathrm{C} 3-\mathrm{C} 6$ bond of approximately $180^{\circ}$ with population parameters 0.762 (3) for $\mathrm{S} 1 A / \mathrm{C} 1 A-\mathrm{C} 5 A$ and 0.238 (3) for $\mathrm{S} 1 B / \mathrm{C} 1 B-\mathrm{C} 5 B$ (Fig. 1). The configuration of the $\mathrm{C} 11=\mathrm{N} 10$ bond can be described as $E$ [the N9-N10-C11-C12 torsion

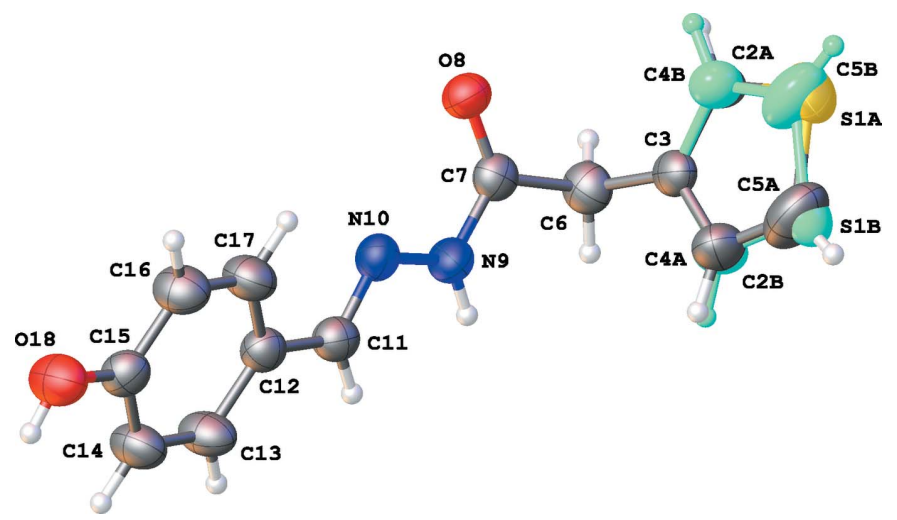

Figure 1

A view of the molecular structure of (3a), with atom labels and displacement ellipsoids drawn at the $50 \%$ probability level. The minordisorder component is shown in light green.

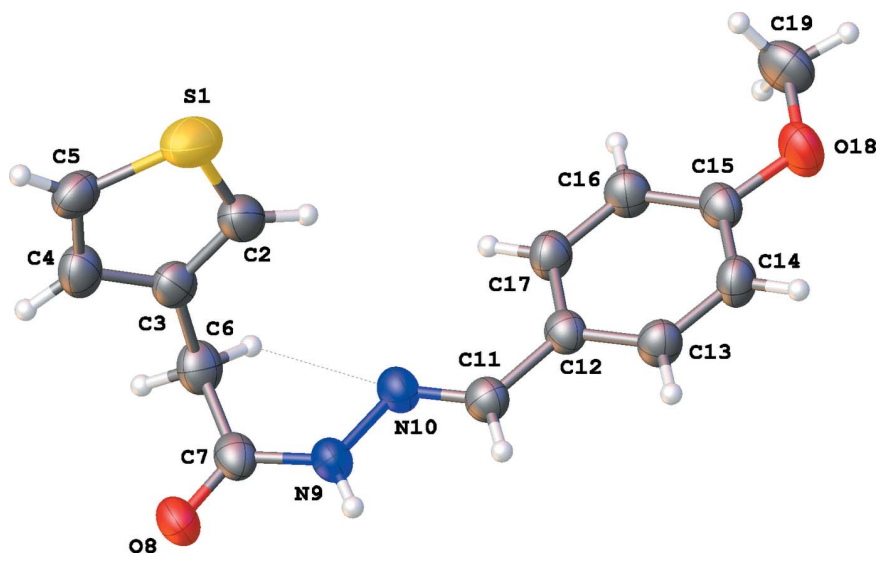

Figure 2

The molecular structure of (3b) with atom labels and $50 \%$ probability displacement ellipsoids.
Table 1

Hydrogen-bond geometry $\left(\AA,^{\circ}\right)$ for (3a).

$\mathrm{Cg} 3$ is the centroid of the $\mathrm{C} 12-\mathrm{C} 17$ phenyl ring.

\begin{tabular}{lllll}
\hline$D-\mathrm{H} \cdots A$ & $D-\mathrm{H}$ & $\mathrm{H} \cdots A$ & $D \cdots A$ & $D-\mathrm{H} \cdots A$ \\
\hline $\mathrm{N} 9-\mathrm{H} 9 \cdots \mathrm{O}^{\mathrm{i}}$ & 0.86 & 2.12 & $2.953(2)$ & 162 \\
$\mathrm{O} 18-\mathrm{H} 18 \cdots \mathrm{O} 8^{\text {ii }}$ & 0.82 & 1.97 & $2.782(2)$ & 169 \\
$\mathrm{C} 2 A-\mathrm{H} 2 A \cdots \mathrm{O} 8^{\text {iii }}$ & 0.93 & 2.57 & $3.439(7)$ & 155 \\
$\mathrm{C} 13-\mathrm{H} 13 \cdots C g 3^{\text {iv }}$ & 0.93 & 2.89 & $3.818(3)$ & 176 \\
\hline
\end{tabular}

Symmetry codes: (i) $-x+\frac{1}{2}, y+\frac{1}{2}, z$; (ii) $x-\frac{1}{2}, y,-z+\frac{1}{2}$; (iii) $-x+1,-y+1,-z+1$; (iv) $-x, y+\frac{1}{2},-z+\frac{1}{2}$.

angle is $\left.174.82(16)^{\circ}\right]$. The torsion angle $\mathrm{C} 7-\mathrm{N} 9-\mathrm{N} 10-\mathrm{C} 11$ of $177.10(18)^{\circ}$ indicates that the conformation around the $\mathrm{N} 9-\mathrm{N} 10$ bond is $+a p$. The molecule is twisted about the C6$\mathrm{C} 7$ bond with a dihedral angle of $84.0(3)^{\circ}$ between the thiophene and benzene rings $\left[87.0(9)^{\circ}\right.$ for $\left.\mathrm{S} 1 B / \mathrm{C} 1 B-\mathrm{C} 5 B\right]$.

The methoxy derivative (3b) (Fig. 2) crystallizes in the triclinic space group $P \overline{1}$. Compared to (3a), the central part of (3b) displays a similar $+a p$ conformation around the N9-N10 bond and an $E$ configuration of the $\mathrm{C} 11=\mathrm{N} 10$ bond, as illustrated by the torsion angles $\mathrm{C} 7-\mathrm{N} 9-\mathrm{N} 10-\mathrm{C} 11\left[177.8(2)^{\circ}\right]$ and $\mathrm{N} 9-\mathrm{N} 10-\mathrm{C} 11-\mathrm{C} 12 \quad\left[179.26(19)^{\circ}\right]$. However, the conformation about the two other bonds, $\mathrm{C} 6-\mathrm{C} 7$ and especially $\mathrm{C} 7-\mathrm{N} 9$, in the linker between both rings is different. The torsion angle $\mathrm{C} 3-\mathrm{C} 6-\mathrm{C} 7-\mathrm{N} 9$ is $-101.8(2)^{\circ}$ (or $-a c$ ) for (3a) and $-85.4(3)^{\circ}($ or $-s c)$ for $(3 b)$. As a consequence, in (3b) a short C6-H6 $\cdots \mathrm{N} 10$ interaction occurs (Table 2). In (3a) we observe an $+a p$ conformation [torsion angle $\mathrm{C} 6-\mathrm{C} 7-\mathrm{N} 9-$ $\mathrm{N} 10$ is $167.45(16)^{\circ}$ ], while this is $-s p$ in (3b) [torsion angle $\mathrm{C} 6-\mathrm{C} 7-\mathrm{N} 9-\mathrm{N} 10$ is $\left.-5.8(3)^{\circ}\right]$. The dihedral angle between the thiophene and phenyl rings is $85.89(12)^{\circ}$, in the same order as for (3a).

\section{Supramolecular features}

In the crystal, molecules of (3a) are connected by N9H9 . O O 8 [symmetry code: (i) $-x+\frac{1}{2}, y+\frac{1}{2}, z$ ] hydrogen bonds, resulting in the formation of chains in the $b$-axis direction with a $C_{1}^{1}(4)$ graph-set motif (Fig. 3, Table 1). In addition, chains

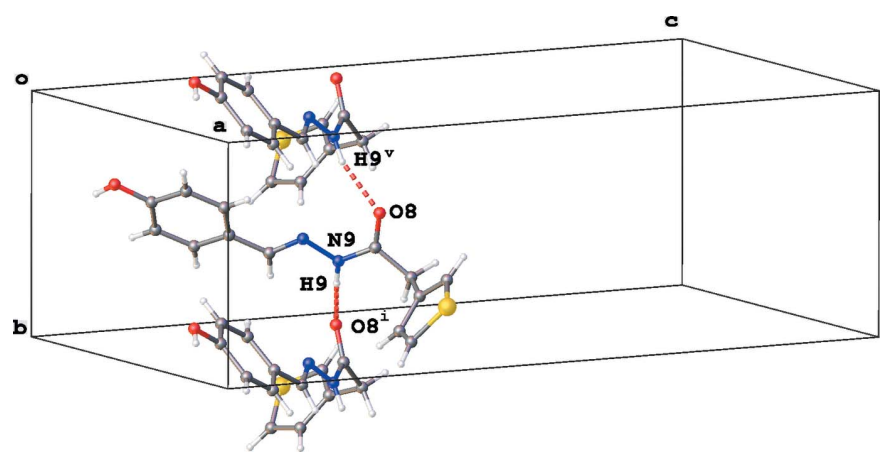

Figure 3

Part of the crystal structure of (3a), showing the chain formation through $\mathrm{N}-\mathrm{H} \cdots \mathrm{O}$ interactions (red dashed lines) along the $b$-axis direction. The minor disorder component is not shown. Symmetry codes: (i) $-x+\frac{1}{2}, y+\frac{1}{2}$, $z ;(\mathrm{v})-x+\frac{1}{2}, y-\frac{1}{2}, z$. 
Table 2

Hydrogen-bond geometry $\left(\AA,^{\circ}\right)$ for (3b).

$C g 1$ is the centroid of the $\mathrm{S} 1 / \mathrm{C} 1-\mathrm{C} 5$ thiophene ring.

\begin{tabular}{lllll}
\hline$D-\mathrm{H} \cdots A$ & $D-\mathrm{H}$ & $\mathrm{H} \cdots A$ & $D \cdots A$ & $D-\mathrm{H} \cdots A$ \\
\hline $\mathrm{N} 9-\mathrm{H} 9 \cdots \mathrm{O} 8^{\mathrm{i}}$ & 0.86 & 2.08 & $2.935(3)$ & 179 \\
$\mathrm{C} 6-\mathrm{H} 6 A \cdots \mathrm{N} 10$ & 0.97 & 2.44 & $2.782(3)$ & 100 \\
$\mathrm{C} 13-\mathrm{H} 13 \cdots C g 1^{\text {ii }}$ & 0.93 & 2.68 & $3.611(2)$ & 179 \\
\hline
\end{tabular}

Symmetry codes: (i) $-x,-y+2,-z+1$; (ii) $-x+1,-y+2,-z+1$.

with a $C_{1}^{1}(11)$ graph-set motif running along the $a$-axis direction are formed by $\mathrm{O} 18-\mathrm{H} 18 \cdots \mathrm{O} 8^{\mathrm{ii}}$ [symmetry code: (ii) $x-\frac{1}{2}$, $y,-z+\frac{1}{2}$ ] hydrogen bonds (Fig. 4, Table 1). Two weaker interactions are present in the packing: a $\mathrm{C}-\mathrm{H} \cdots \mathrm{O}$ and $\mathrm{C}-$ $\mathrm{H} \cdots \pi$ (phenyl) interaction (for details see Table 1).

Replacing the $-\mathrm{OH}$ group in (3a) by an -OMe group in (3b) changes the hydrogen-bonding pattern. The crystal packing of (3b) is now characterized by the presence of two different inversion dimers. The first type, with an $R_{2}^{2}(8)$ graph-set motif, is formed by $\mathrm{N} 9-\mathrm{H} 9 \cdots \mathrm{O} 8^{\mathrm{i}}$ [symmetry code: (i) $-x,-y+2$, $-z+1$ ] hydrogen bonds (Fig. 5, Table 2). The second one involves $\mathrm{C} 13-\mathrm{H} 13 \cdots \pi$ (thiophene) interactions (Fig. 6, Table 2).

No voids or $\pi-\pi$ stackings are observed in the crystal packing of (3a) and (3b).

\section{Database survey}

A search of the Cambridge Structural Database (CSD, Version 5.40, update of May 2019; Groom et al., 2016) for the

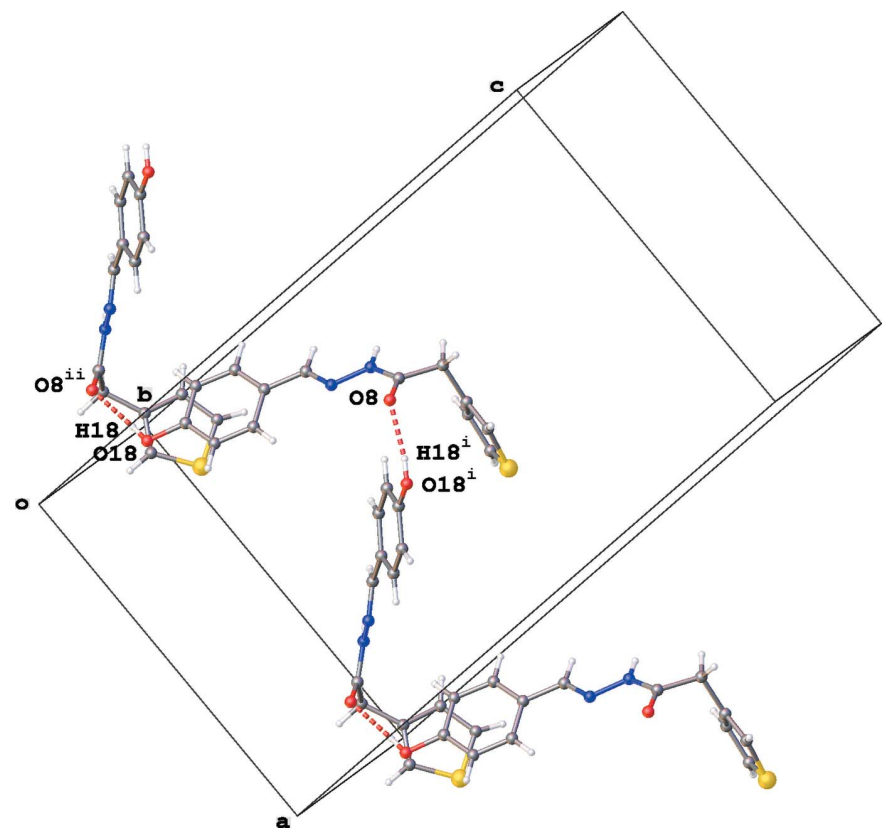

Figure 4

Part of the crystal structure of (3a), illustrating the chain formation through $\mathrm{O}-\mathrm{H} \cdots \mathrm{O}$ interactions (red dashed lines) along the $a$-axis direction. The minor disorder component is not shown. Symmetry codes: (i) $x+\frac{1}{2}, y,-z+\frac{1}{2}$; (ii) $x-\frac{1}{2}, y,-z+\frac{1}{2}$.

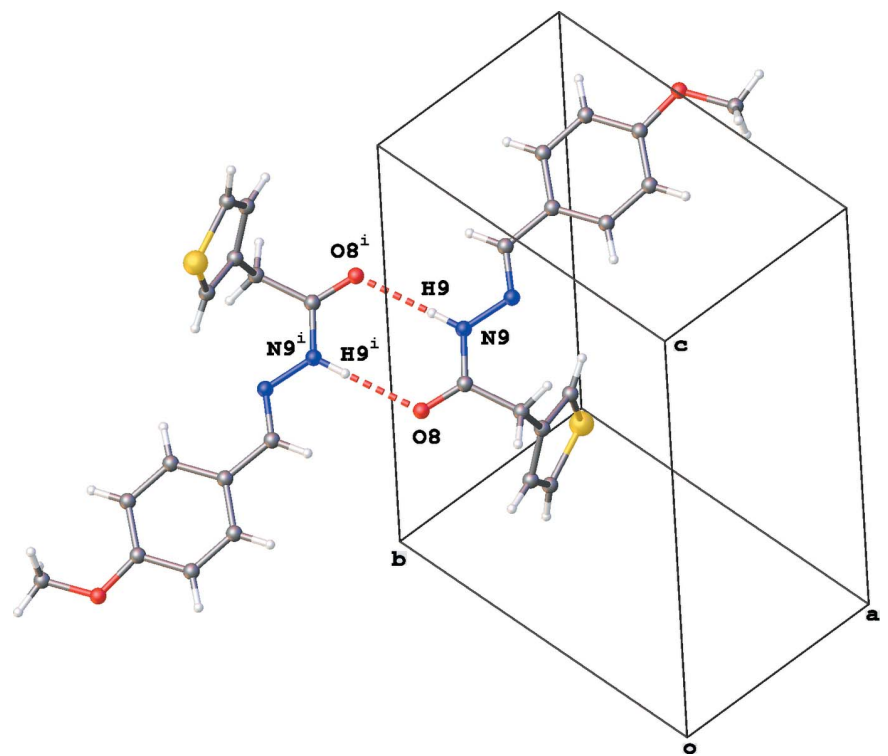

Figure 5

A partial packing diagram of (3b), showing dimer formation through $\mathrm{N}-$ $\mathrm{H} \cdots \mathrm{O}$ interactions (red dashed lines). Symmetry code: (i) $-x,-y+2$, $-z+1$.

central linker between the two rings in the title compound, $\mathrm{C}-\mathrm{CH}_{2}-\mathrm{C}(=\mathrm{O})-\mathrm{NH}-\mathrm{N}=\mathrm{CH}-\mathrm{C}$ (Fig. 7a), resulted in

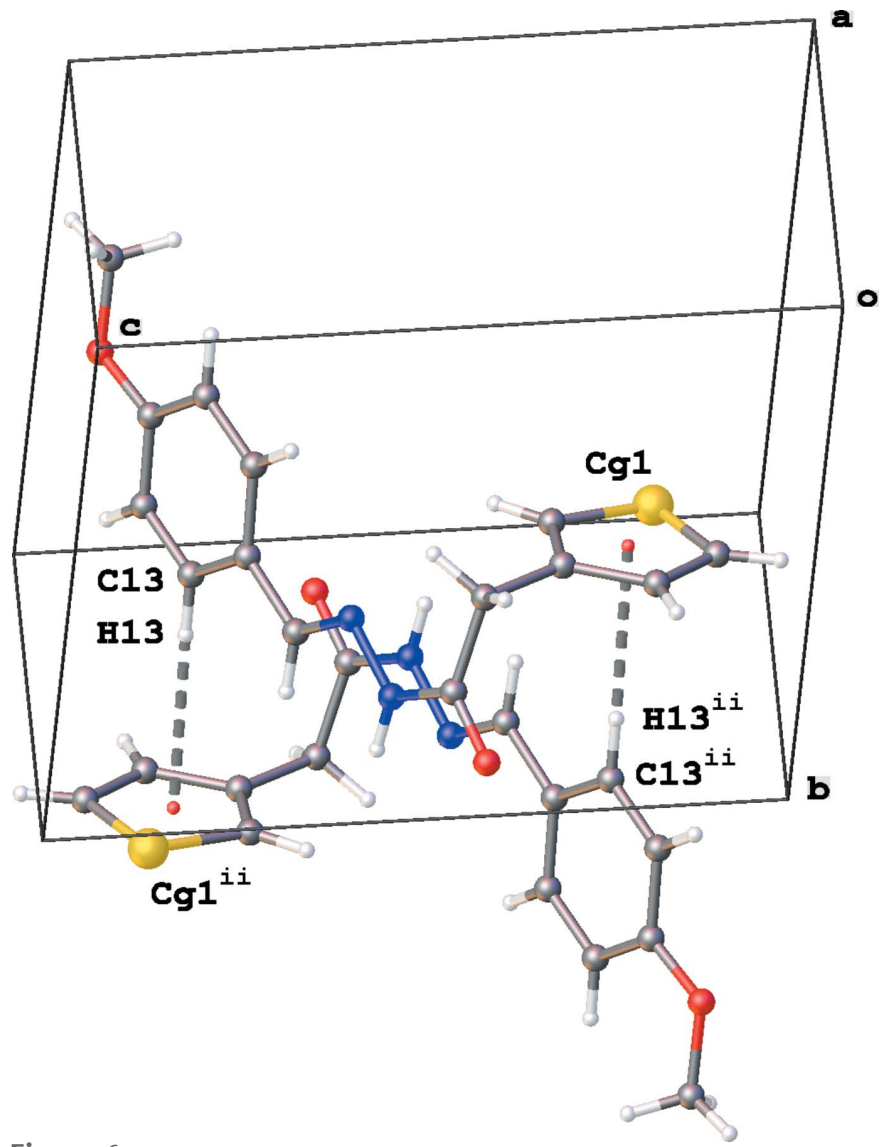

Figure 6

A partial packing diagram of (3b), illustrating the dimer formation through $\mathrm{C}-\mathrm{H} \cdots \pi$ interactions (gray dashed lines). $C g 1$ is the centroid of the S1/C2-C5 thiophene ring. Symmetry code: (ii) $-x+1,-y+2,-z+1$. 
(a)<smiles>C/C=N/NC(=O)CC</smiles>

(b)

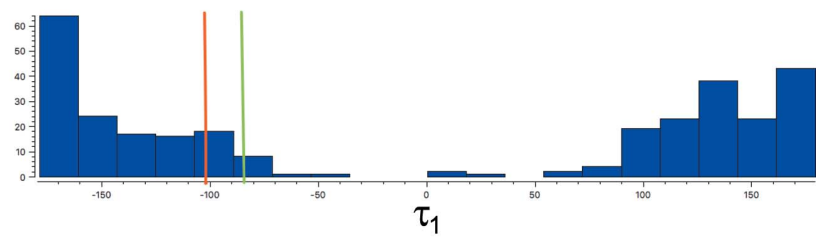

(c)

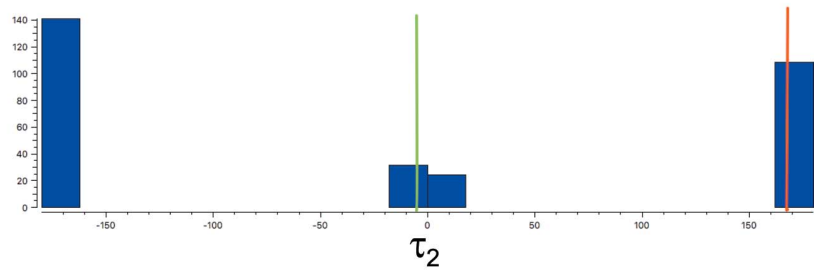

(d)

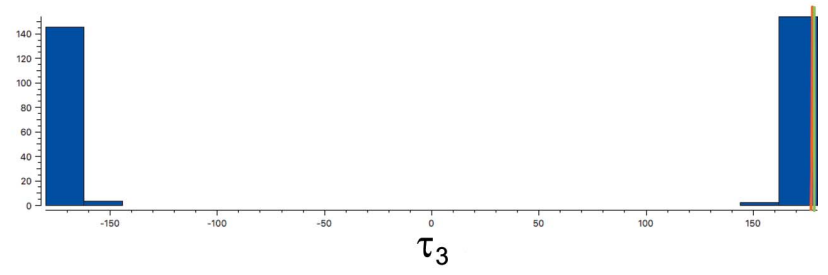

(e)

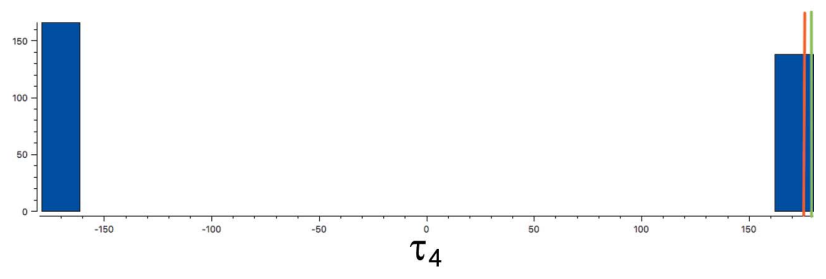

Figure 7

(a) Fragment used for a search in the CSD. (b)-(e) Histograms of torsion angles $\tau_{1}, \tau_{2}, \tau_{3}$ and $\tau_{4}$, respectively. The vertical red and green lines show the torsion angles observed in title compounds (3a) and (3b), respectively.

137 hits. Histograms of the distribution of the four torsion angles $\tau_{1}-\tau_{4}$ along the linker backbone are shown in Fig. $7 b-e$ [the red and green lines depict the torsion angles for title compounds (3a) and (3b), respectively]. The histogram of $\tau_{1}$ reflects a wide spread with a preference for the $-a p /+a p$ conformation, followed by the $-s c /+s c$ conformation and only a few entries in the remaining regions. In the case of torsion angle $\tau_{2}$, two regions are preferred: $-a p /+a p$ [for the majority of the entries and similar to (3a)] and $-s p /+s p$ [similar to (3b)]. Torsion angles $\tau_{3}$ and $\tau_{4}$ show both a narrow spread in the region $-a p /+a p$.

\section{Hirshfeld surface analysis}

The Hirshfeld surface analysis (Spackman \& Jayatilaka, 2009) and the associated two-dimensional fingerprint plots (McKinnon et al., 2007) were performed using CrystalExplorer (Turner et al., 2017). The Hirshfeld surfaces of compounds (3a) and (3b) mapped over $d_{\text {norm }}$ are given in Fig. 8.
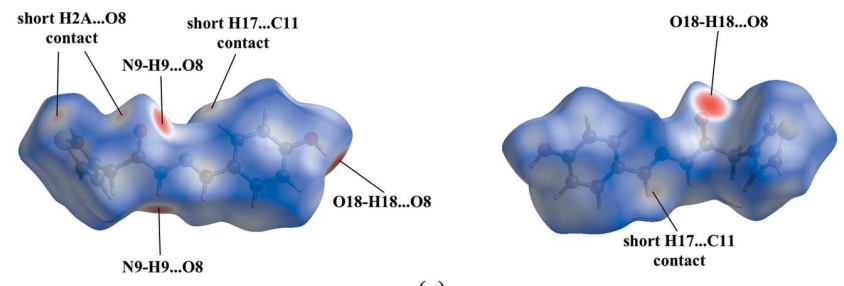

(a)

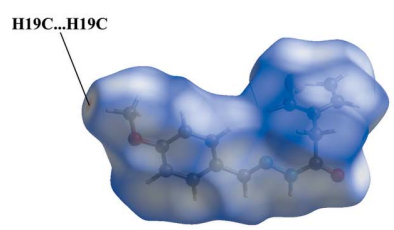

(b)

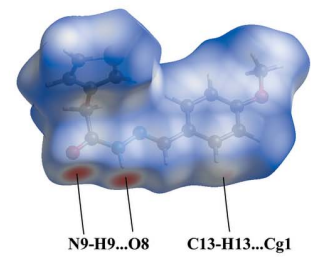

Figure 8

The Hirshfeld surface mapped over $d_{\text {norm }}$ for (a) compound (3a) in the range -0.6166 to 1.1782 a.u., and $(b)$ compound ( $3 b)$ in the range -0.5274 to 1.2642 a.u.

The bright-red spots in Fig. $8 a$ near atoms $\mathrm{O} 8$ and $\mathrm{N} 9$ illustrate the $\mathrm{N} 9-\mathrm{H} 9 \cdots \mathrm{O} 8$ hydrogen bond, and near atoms $\mathrm{O} 8$ and $\mathrm{O} 18$ the $\mathrm{O} 18-\mathrm{H} 18 \cdots \mathrm{O} 8$ hydrogen bond. The faintred spots near atoms $\mathrm{O} 8$ and $\mathrm{H} 2 A$, and $\mathrm{C} 11$ and $\mathrm{H} 17$ refer to short contacts in the crystal packing of (3a). The most significant contributions to the Hirshfeld surface are from $\mathrm{H} \cdots \mathrm{H}$ $(30.5 \%), \mathrm{C} \cdots \mathrm{H} / \mathrm{H} \cdots \mathrm{C}(26.1 \%), \mathrm{O} \cdots \mathrm{H} / \mathrm{H} \cdots \mathrm{O}(18.6 \%)$ and S $\cdots \mathrm{H} / \mathrm{H} \cdots \mathrm{S}(10.7 \%)$ contacts.

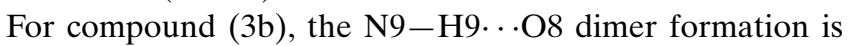
viewed as the bright-red spots near atoms O8 and N9 in Fig. $8 b$. The faint-red spots near atoms $\mathrm{H} 19 C$ and $\mathrm{H} 13$ are indicative for a short $\mathrm{H} 19 \mathrm{C} \cdots \mathrm{H} 19 \mathrm{C}$ contact and the $\mathrm{C} 13-$ H13 $\cdots \pi$ (thiophene) interaction. The most significant contributions to the Hirshfeld surface are from $\mathrm{H} \cdots \mathrm{H}(40.6 \%)$, $\mathrm{C} \cdots \mathrm{H} / \mathrm{H} \cdots \mathrm{C}(22.2 \%), \mathrm{O} \cdots \mathrm{H} / \mathrm{H} \cdots \mathrm{O}(15.1 \%)$ and $\mathrm{S} \cdots \mathrm{H} /$ $\mathrm{H}$...S $(12.5 \%)$ contacts.

\section{Synthesis and crystallization}

The reaction scheme to synthesize the title compounds, (3a) and (3b), is given in Fig. 9.

Methyl 2-(thiophen-3-yl)acetate (1) and 2-(thiophen-3yl)acetohydrazide (2) were synthesized according to our previous research (Vu et al., 2017).

Synthesis of $N^{\prime}$-[1-(4-hydroxyphenyl)benzylidene]-2(thiophen-3-yl)acetohydrazide:

Compound (2) $(3 \mathrm{mmol})$ and the appropriate benzaldehyde derivatives $(6 \mathrm{mmol})$ with acetic acid $(1.5 \mathrm{~mL})$ in ethanol $(20 \mathrm{~mL})$ were refluxed for $5 \mathrm{~h}$. The reaction mixture was
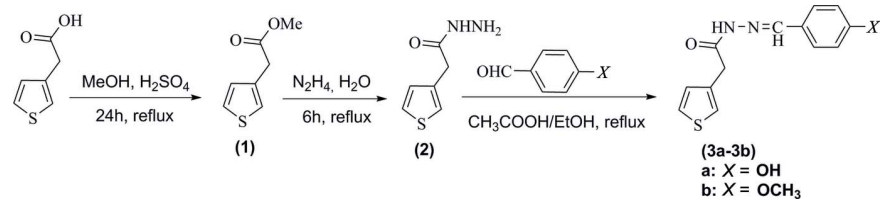

Figure 9

Reaction scheme for the title compounds (3a) and (3b). 
Table 3

Experimental details.

(3a)

Crystal data

Chemical formula

$M_{\text {r }}$

Crystal system, space group

Temperature (K)

$a, b, c(\AA)$

$\alpha, \beta, \gamma\left({ }^{\circ}\right)$

$V\left(\AA^{3}\right)$

$Z$

Radiation type

$\mu\left(\mathrm{mm}^{-1}\right)$

Crystal size (mm)

Data collection

Diffractometer

Absorption correction

$T_{\min }, T_{\max }$

No. of measured, independent and

observed $[I>2 \sigma(I)]$ reflections

$R_{\text {int }}$

$(\sin \theta / \lambda)_{\max }\left(\AA^{-1}\right)$

$\mathrm{C}_{13} \mathrm{H}_{12} \mathrm{~N}_{2} \mathrm{O}_{2} \mathrm{~S}$

260.31

Orthorhombic, $\mathrm{Pbca}$

293

13.0820 (8), 8.0287 (4), 24.0442 (12)

$90,90,90$

$2525.4(2)$

8

Mo $K \alpha$

0.25

$0.35 \times 0.2 \times 0.05$

Rigaku Oxford Diffraction SuperNova, Single source at offset/far, Eos

Multi-scan (CrysAlis PRO; Rigaku OD, 2018)

$0.453,1.000$

$13596,2571,1759$

0.039

0.625

$0.046,0.109,1.07$

2571

178

80

$\mathrm{H}$-atom parameters constrained

$0.19,-0.18$ (3b)

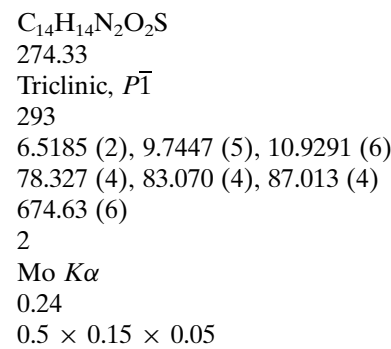

Rigaku Oxford Diffraction SuperNova, Single source at offset/far, Eos

Multi-scan (CrysAlis PRO; Rigaku OD, 2018)

$0.687,1.000$

$13795,2752,2238$

0.027

0.625

$0.051,0.145,1.06$

2752

173

0

$\mathrm{H}$-atom parameters constrained $0.33,-0.38$

$\Delta \rho_{\max }, \Delta \rho_{\min }\left(\mathrm{e} \AA^{-3}\right)$

Computer programs: CrysAlis PRO (Rigaku OD, 2018), SHELXT (Sheldrick, 2015a), SHELXL (Sheldrick, 2015b) and OLEX2 (Dolomanov et al., 2009).

cooled down and the solid product was separated by filtration and purified by recrystallization in ethanol to give the compounds (3a) and (3b).

Data for $N^{\prime}$-[1-(4-hydroxyphenyl)benzylidene]-2-(thiophen-3-yl)acetohydrazide (3a):

White crystals; m.p. $443 \mathrm{~K}$; yield $63 \%$. IR $\left(\mathrm{KBr}, \mathrm{cm}^{-1}\right)$ : 3289 , $3207(\mathrm{NH}), 3050,2874(\mathrm{C}-\mathrm{H}), 1621(\mathrm{C}=\mathrm{O}), 1606(\mathrm{CH}=\mathrm{N})$, $1511(\mathrm{C}=\mathrm{C}) .{ }^{1} \mathrm{H}$ NMR [Bruker XL-500, $500 \mathrm{MHz}, d_{6}-\mathrm{CDCl}_{3}$, $\delta(\mathrm{ppm}), J(\mathrm{~Hz})]: 7.19\left(m, 1 \mathrm{H}, \mathrm{H}^{2}\right), 7.11\left(d, 1 \mathrm{H},{ }^{5} J=5.0, \mathrm{H}^{4}\right)$, $7.25\left(d d, 1 \mathrm{H},{ }^{2} J=3.0,{ }^{4} J=5.0, \mathrm{H}^{5}\right), 4.07\left(s, 2 \mathrm{H}, \mathrm{H}^{6}\right), 9.17(s, 1 \mathrm{H}$, $\left.\mathrm{H}^{8}\right), 7.79\left(s, 1 \mathrm{H}, \mathrm{H}^{9}\right), 7.52\left(d, 2 \mathrm{H}, J=8.5 \mathrm{H}^{11}, \mathrm{H}^{15}\right), 6.87(d, 2 \mathrm{H}$, $\left.J=8.5 \mathrm{H}^{12}, \mathrm{H}^{14}\right), 10.10 / 10.04\left(s, 1 \mathrm{H}, \mathrm{H}^{16}\right) .{ }^{13} \mathrm{C}$ NMR [Bruker $\left.\mathrm{XL}-500,125 \mathrm{MHz}, d_{6}-\mathrm{CDCl}_{3}, \delta(\mathrm{ppm})\right]: 122.3 / 122.4\left(\mathrm{C}^{2}\right), 135.3 /$ $135.4\left(C^{3}\right), 128.7 / 128.8\left(C^{4}\right), 125.4 / 125.8\left(C^{5}\right), 33.6 / 35.9\left(C^{6}\right)$, 165.7/171.4 $\left(C^{7}\right), 146.7\left(C^{9}\right), 143.5\left(C^{10}\right), 128.3 / 128.6\left(C^{11}, C^{15}\right)$, 115.6/116.6 $\left(\mathrm{C}^{12}, \mathrm{C}^{14}\right), \quad 159.6 / 159.2 \quad\left(\mathrm{C}^{13}\right)$. Calculation for $\mathrm{C}_{13} \mathrm{H}_{12} \mathrm{~N}_{2} \mathrm{O}_{2} \mathrm{~S}: M^{[+\mathrm{H}]}=260.9$ au.

Data for N'-[1-(4-methoxyphenyl)benzylidene]-2-(thiophen-3-yl)acetohydrazide (3b):

White crystals, m.p. $431 \mathrm{~K}$, yield $53 \%$. IR $\left(\mathrm{KBr}, \mathrm{cm}^{-1}\right)$ : 3442, $3112(\mathrm{NH}), 3014,2950(\mathrm{C}-\mathrm{H}), 1706(\mathrm{C}=\mathrm{O}), 1617(\mathrm{CH}=\mathrm{N})$, 1558, $1503(\mathrm{C}=\mathrm{C}) .{ }^{1} \mathrm{H}$ NMR [Bruker XL-500, $500 \mathrm{MHz}, d_{6^{-}}$ $\left.\mathrm{CDCl}_{3}, \delta(\mathrm{ppm}), J(\mathrm{~Hz})\right]: 7.22\left(m, 1 \mathrm{H}, \mathrm{H}^{2}\right) ; 7.12\left(m, 1 \mathrm{H}, \mathrm{H}^{4}\right)$; $7.26\left(d d, 1 \mathrm{H},{ }^{2} J=3.0,{ }^{5} J=5.0, \mathrm{H}^{5}\right) ; 4.11\left(s, 2 \mathrm{H}, \mathrm{H}^{6}\right) ; 8.97(s, 1 \mathrm{H}$, $\left.\mathrm{H}^{8}\right) ; 7.69\left(s, 1 \mathrm{H}, \mathrm{H}^{9}\right) ; 7.61\left(d, 2 \mathrm{H}, J=8.5, \mathrm{H}^{11}, \mathrm{H}^{15}\right) ; 6.94(d, 2 \mathrm{H}$, $\left.J=8.5, \mathrm{H}^{12}, \mathrm{H}^{14}\right) ; 3.85\left(m, 3 \mathrm{H}, \mathrm{H}^{16}\right) .{ }^{13} \mathrm{C}$ NMR [Bruker XL500, $\left.125 \mathrm{MHz}, d_{6}-\mathrm{CDCl}_{3}, \delta(\mathrm{ppm})\right]: 122.8\left(\mathrm{C}^{2}\right), 134.4\left(\mathrm{C}^{3}\right)$,
$129.3\left(C^{4}\right), 125.4\left(C^{5}\right), 34.3\left(C^{6}\right), 172.9\left(C^{7}\right), 143.6\left(C^{9}\right), 126.4$ $\left(\mathrm{C}^{10}\right), 128.8\left(\mathrm{C}^{11}, \mathrm{C}^{15}\right), 114.3\left(\mathrm{C}^{12}, \mathrm{C}^{14}\right), 161.3\left(\mathrm{C}^{13}\right), 55.4\left(\mathrm{C}^{16}\right)$. Calculation for $\mathrm{C}_{14} \mathrm{H}_{14} \mathrm{~N}_{2} \mathrm{O}_{2} \mathrm{~S}: M^{[+\mathrm{H}]}=274.9 \mathrm{au}$.

\section{Refinement details}

Crystal data, data collection and structure refinement details are summarized in Table 3. All $\mathrm{H}$ atoms were placed in idealized positions and refined in riding mode, with $U_{\text {iso }}(\mathrm{H})$ values assigned as $1.2 U_{\text {eq }}$ of the parent atoms (1.5 times for methyl groups), with $\mathrm{C}-\mathrm{H}$ distances of 0.93 (aromatic), 0.96 $\left(\mathrm{CH}_{3}\right)$ and $0.97 \AA\left(\mathrm{CH}_{2}\right), \mathrm{N}-\mathrm{H}$ distances of $0.86 \AA$ and $\mathrm{O}-\mathrm{H}$ distances of $0.82 \AA$ (rotating $\mathrm{OH}$ ). In (3a), the thiophene ring is disordered over two positions [population parameters 0.762 (3) and 0.238 (3)] and was refined with restraints for the bond lengths and angles in the ring. The anisotropic temperature factors for atoms S1, C2, C4 and C5 in both orientations were constrained to be equal. In the final cycles of refinement, four and two outliers were omitted for (3a) and (3b), respectively.

\section{Acknowledgements}

LVM thanks the Hercules Foundation for supporting the purchase of the diffractometer through project AKUL/09/ 0035 . 


\section{References}

Bharti, S. K., Nath, G., Tilak, R. \& Singh, S. K. (2010). Eur. J. Med. Chem. 45, 651-660.

Dolomanov, O. V., Bourhis, L. J., Gildea, R. J., Howard, J. A. K. \& Puschmann, H. (2009). J. Appl. Cryst. 42, 339-341.

El-Sayed, H. A., Moustafa, A. H., El-Moneim, M. A., Awad, H. M. \& Esmat, A. (2018). J. Pharm. Appl. Chem. 4, 125-131.

Groom, C. R., Bruno, I. J., Lightfoot, M. P. \& Ward, S. C. (2016). Acta Cryst. B72, 171-179.

Loncle, C., Brunel, J. M., Vidal, N., Dherbomez, M. \& Letourneux, Y. (2004). Eur. J. Med. Chem. 39, 1067-1071.

McKinnon, J. J., Jayatilaka, D. \& Spackman, M. A. (2007). Chem. Commun. pp. 3814-3816.

Nguyen, N. L., Tran, T. D., Nguyen, T. C., Duong, K. L., Pfleger, J. \& Vu, Q. T. (2016). Vietnam. J. Chem. 54, 259-263.

Papakonstantinou-Garoufalias, S., Pouli, N., Marakos, P. \& Chytyroglou-Ladas, A. (2002). Farmaco, 57, 973-977.
Rigaku OD (2018). CrysAlis PRO. Rigaku Oxford Diffraction, Yarnton, England.

Sheldrick, G. M. (2015a). Acta Cryst. A71, 3-8.

Sheldrick, G. M. (2015). Acta Cryst. C71, 3-8.

Spackman, M. A. \& Jayatilaka, D. (2009). CrystEngComm, 11, 1932.

Turner, M. J., McKinnon, J. J., Wolff, S. K., Grimwood, D. J., Spackman, P. R., Jayatilaka, D. \& Spackman, M. A. (2017). CrystalExplorer 17. University of Western Australia. http:// hirshfeldsurface.net

Vu, Q. T., Nguyen, N. L., Duong, K. L. \& Pfleger, J. (2016). Vietnam. J. Chem. 54, 730-735.

Vu Quoc, T., Nguyen Ngoc, L., Nguyen Tien, C., Thang Pham, C. \& Van Meervelt, L. (2017). Acta Cryst. E73, 901-904.

Wardakhan, W. W., Eid, E.-S. N. N \& Mohareb, R. M. (2013). Acta Pharm. 63, 45-57.

Yadav, M., Sinha, R. R., Kumar, S., Bahadur, I. \& Ebenso, E. E. (2015). J. Mol. Liq. 208, 322-332. 


\section{supporting information}

Acta Cryst. (2019). E75, 1090-1095 [https://doi.org/10.1107/S2056989019008892]

Crystal structure of two N'-(1-phenylbenzylidene)-2-(thiophen-3yl)acetohydrazides

Trung Vu Quoc, Linh Nguyen Ngoc, Duong Tran Thi Thuy, Manh Vu Quoc, Thien Vuong Nguyen, Yen Oanh Doan Thi and Luc Van Meervelt

Computing details

For both structures, data collection: CrysAlis PRO (Rigaku OD, 2018); cell refinement: CrysAlis PRO (Rigaku OD, 2018); data reduction: CrysAlis PRO (Rigaku OD, 2018); program(s) used to solve structure: SHELXT (Sheldrick, 2015a); program(s) used to refine structure: SHELXL (Sheldrick, 2015b); molecular graphics: OLEX2 (Dolomanov et al., 2009); software used to prepare material for publication: OLEX2 (Dolomanov et al., 2009).

$N^{\prime}$-[1-(4-Hydroxyphenyl)benzylidene]-2-(thiophen-3-yl)acetohydrazide (3a)

\section{Crystal data}

$\mathrm{C}_{13} \mathrm{H}_{12} \mathrm{~N}_{2} \mathrm{O}_{2} \mathrm{~S}$

$M_{r}=260.31$

Orthorhombic, $\mathrm{Pbca}$

$a=13.0820(8) \AA$

$b=8.0287(4) \AA$

$c=24.0442(12) \AA$

$V=2525.4(2) \AA^{3}$

$Z=8$

$F(000)=1088$

Data collection

Rigaku Oxford Diffraction SuperNova, Single source at offset/far, Eos diffractometer

Radiation source: micro-focus sealed X-ray tube, SuperNova (Mo) X-ray Source

Mirror monochromator

Detector resolution: 15.9631 pixels $\mathrm{mm}^{-1}$

$\omega$ scans

Absorption correction: multi-scan

(CrysAlis PRO; Rigaku OD, 2018)

Refinement

Refinement on $F^{2}$

Least-squares matrix: full

$R\left[F^{2}>2 \sigma\left(F^{2}\right)\right]=0.046$

$w R\left(F^{2}\right)=0.109$

$S=1.07$

2571 reflections

178 parameters
$D_{\mathrm{x}}=1.369 \mathrm{Mg} \mathrm{m}^{-3}$

Mo $K \alpha$ radiation, $\lambda=0.71073 \AA$

Cell parameters from 3446 reflections

$\theta=3.1-23.7^{\circ}$

$\mu=0.25 \mathrm{~mm}^{-1}$

$T=293 \mathrm{~K}$

Plate, white

$0.35 \times 0.2 \times 0.05 \mathrm{~mm}$

$T_{\min }=0.453, T_{\max }=1.000$

13596 measured reflections

2571 independent reflections

1759 reflections with $I>2 \sigma(I)$

$R_{\text {int }}=0.039$

$\theta_{\max }=26.4^{\circ}, \theta_{\min }=3.1^{\circ}$

$h=-16 \rightarrow 15$

$k=-9 \rightarrow 10$

$l=-28 \rightarrow 30$

80 restraints

Hydrogen site location: inferred from neighbouring sites

$\mathrm{H}$-atom parameters constrained

$w=1 /\left[\sigma^{2}\left(F_{\mathrm{o}}^{2}\right)+(0.0334 P)^{2}+0.6755 P\right]$

where $P=\left(F_{\mathrm{o}}{ }^{2}+2 F_{\mathrm{c}}{ }^{2}\right) / 3$

$(\Delta / \sigma)_{\max }<0.001$ 
$\Delta \rho_{\max }=0.19 \mathrm{e} \AA^{-3}$

$\Delta \rho_{\min }=-0.18$ e $\AA^{-3}$
Extinction correction: SHELXL (Sheldrick, 2015b), $\mathrm{Fc}^{*}=\mathrm{kFc}\left[1+0.001 \mathrm{xFc}^{2} \lambda^{3} / \sin (2 \theta)\right]^{-1 / 4}$ Extinction coefficient: $0.0022(6)$

Special details

Geometry. All esds (except the esd in the dihedral angle between two 1.s. planes) are estimated using the full covariance matrix. The cell esds are taken into account individually in the estimation of esds in distances, angles and torsion angles; correlations between esds in cell parameters are only used when they are defined by crystal symmetry. An approximate (isotropic) treatment of cell esds is used for estimating esds involving l.s. planes.

Fractional atomic coordinates and isotropic or equivalent isotropic displacement parameters $\left(\AA^{2}\right)$

\begin{tabular}{|c|c|c|c|c|c|}
\hline & $x$ & $y$ & $z$ & $U_{\text {iso }} * / U_{\text {eq }}$ & Occ. $(<1)$ \\
\hline S1A & $0.67164(8)$ & $0.8283(2)$ & $0.43607(8)$ & 0.0718 (4) & $0.762(3)$ \\
\hline S1B & $0.6152(4)$ & $0.9867(10)$ & $0.3990(3)$ & $0.0718(4)$ & $0.238(3)$ \\
\hline $\mathrm{C} 2 \mathrm{~A}$ & $0.5667(3)$ & $0.7484(7)$ & $0.4673(3)$ & $0.0542(12)$ & $0.762(3)$ \\
\hline $\mathrm{H} 2 \mathrm{~A}$ & 0.568185 & 0.659897 & 0.492216 & $0.065 *$ & $0.762(3)$ \\
\hline $\mathrm{C} 2 \mathrm{~B}$ & $0.4941(11)$ & $0.970(3)$ & $0.4213(16)$ & $0.0542(12)$ & $0.238(3)$ \\
\hline $\mathrm{H} 2 \mathrm{~B}$ & 0.443026 & 1.047111 & 0.413833 & $0.065^{*}$ & $0.238(3)$ \\
\hline C3 & $0.47998(16)$ & $0.8287(2)$ & $0.45167(8)$ & $0.0449(5)$ & \\
\hline $\mathrm{C} 4 \mathrm{~A}$ & $0.5018(5)$ & $0.9582(10)$ & $0.4141(5)$ & $0.062(2)$ & $0.762(3)$ \\
\hline $\mathrm{H} 4 \mathrm{~A}$ & 0.451169 & 1.026238 & 0.399205 & $0.074^{*}$ & $0.762(3)$ \\
\hline C4B & $0.5719(9)$ & $0.741(3)$ & $0.4587(11)$ & $0.062(2)$ & $0.238(3)$ \\
\hline H4B & 0.575924 & 0.640913 & 0.478166 & $0.074^{*}$ & $0.238(3)$ \\
\hline $\mathrm{C} 5 \mathrm{~A}$ & $0.6056(6)$ & $0.9770(11)$ & 0.4008 (4) & $0.100(3)$ & $0.762(3)$ \\
\hline $\mathrm{H} 5 \mathrm{~A}$ & 0.633233 & 1.056338 & 0.376954 & $0.120^{*}$ & $0.762(3)$ \\
\hline $\mathrm{C} 5 \mathrm{~B}$ & $0.6580(10)$ & $0.818(2)$ & $0.4337(11)$ & $0.100(3)$ & $0.238(3)$ \\
\hline H5B & 0.725438 & 0.781636 & 0.435909 & $0.120^{*}$ & $0.238(3)$ \\
\hline C6 & $0.37396(16)$ & $0.7786(3)$ & $0.47035(8)$ & $0.0506(6)$ & \\
\hline H6A & 0.377254 & 0.726825 & 0.506761 & $0.061^{*}$ & \\
\hline H6B & 0.330528 & 0.876200 & 0.472922 & $0.061 *$ & \\
\hline C7 & $0.33041(15)$ & 0.6577 (3) & $0.42864(8)$ & $0.0419(5)$ & \\
\hline O8 & $0.36037(11)$ & $0.51138(17)$ & $0.42613(5)$ & 0.0476 (4) & \\
\hline N9 & $0.26256(13)$ & $0.7198(2)$ & $0.39247(6)$ & 0.0455 (4) & \\
\hline H9 & 0.236595 & 0.816955 & 0.397669 & $0.055^{*}$ & \\
\hline N10 & $0.23452(13)$ & $0.6255(2)$ & $0.34641(6)$ & 0.0441 (4) & \\
\hline C11 & $0.16622(15)$ & $0.6908(2)$ & $0.31536(8)$ & $0.0444(5)$ & \\
\hline H11 & 0.135667 & 0.790093 & 0.326364 & $0.053^{*}$ & \\
\hline $\mathrm{C} 12$ & $0.13478(16)$ & $0.6139(2)$ & $0.26302(8)$ & $0.0428(5)$ & \\
\hline $\mathrm{C} 13$ & $0.04946(18)$ & $0.6712(3)$ & $0.23499(9)$ & $0.0553(6)$ & \\
\hline H13 & 0.012178 & 0.759047 & 0.249952 & $0.066^{*}$ & \\
\hline C14 & $0.01803(18)$ & $0.6010(3)$ & $0.18517(9)$ & $0.0561(6)$ & \\
\hline H14 & -0.040209 & 0.640454 & 0.167331 & $0.067^{*}$ & \\
\hline $\mathrm{C} 15$ & $0.07373(17)$ & $0.4720(2)$ & $0.16220(8)$ & $0.0462(5)$ & \\
\hline $\mathrm{C} 16$ & $0.16018(17)$ & $0.4149(3)$ & $0.18911(9)$ & $0.0562(6)$ & \\
\hline H16 & 0.198233 & 0.328679 & 0.173641 & $0.067^{*}$ & \\
\hline $\mathrm{C} 17$ & $0.19021(17)$ & $0.4850(3)$ & $0.23870(9)$ & $0.0538(6)$ & \\
\hline H17 & 0.248657 & 0.445441 & 0.256329 & $0.065^{*}$ & \\
\hline $\mathrm{O} 18$ & $0.04708(13)$ & $0.39577(19)$ & $0.11361(6)$ & $0.0620(5)$ & \\
\hline
\end{tabular}




$\begin{array}{llll}\mathrm{H} 18 & -0.003809 & 0.441085 & 0.100663\end{array}$

Atomic displacement parameters $\left(\AA^{2}\right)$

\begin{tabular}{|c|c|c|c|c|c|c|}
\hline & $U^{11}$ & $U^{22}$ & $U^{33}$ & $U^{12}$ & $U^{13}$ & $U^{23}$ \\
\hline S1A & $0.0493(6)$ & $0.0807(8)$ & $0.0853(7)$ & $0.0003(5)$ & -0.0078 & -0.0050 \\
\hline S1B & $0.0493(6)$ & $0.0807(8)$ & $0.0853(7)$ & $0.0003(5)$ & $-0.0078(5)$ & -0.0050 \\
\hline $\mathrm{C} 2 \mathrm{~A}$ & $0.065(2)$ & $0.051(2)$ & $0.047(3)$ & $0.0092(16)$ & $-0.0120(16)$ & $0.0016(17)$ \\
\hline $\mathrm{C} 2 \mathrm{~B}$ & $0.065(2)$ & $0.051(2)$ & $0.047(3)$ & $0.0092(16)$ & $-0.0120(16)$ & $0.0016(17)$ \\
\hline $\mathrm{C} 3$ & $0.0513(14)$ & 0.0403 (11) & $0.0430(11)$ & $-0.0013(10)$ & $-0.0106(10)$ & $-0.0057(9)$ \\
\hline $\mathrm{C} 4 \mathrm{~A}$ & $0.060(3)$ & $0.053(3)$ & $0.072(6)$ & $-0.0011(18)$ & $-0.008(2)$ & $0.015(3)$ \\
\hline C4B & $0.060(3)$ & $0.053(3)$ & $0.072(6)$ & $-0.0011(18)$ & $-0.008(2)$ & $0.015(3)$ \\
\hline C5A & $0.132(6)$ & $0.063(3)$ & $0.103(4)$ & $-0.025(3)$ & $-0.011(3)$ & $0.021(2)$ \\
\hline C5B & $0.132(6)$ & $0.063(3)$ & $0.103(4)$ & -0.025 & $-0.011(3)$ & $0.021(2)$ \\
\hline C6 & $0.0551(15)$ & $0.0539(13)$ & $0.0427(11)$ & $0.0014(11)$ & $-0.0020(10)$ & $-0.0088(10)$ \\
\hline $\mathrm{C} 7$ & 0.0408 (13) & $0.0444(12)$ & 0.0405 (11) & $-0.0017(9)$ & $0.0056(9)$ & $-0.0002(9)$ \\
\hline $\mathrm{O} 8$ & $0.0533(10)$ & $0.0400(8)$ & 0.0495 (8) & $0.0012(7)$ & $-0.0023(7)$ & $0.0005(6)$ \\
\hline N9 & $0.0489(11)$ & $0.0370(9)$ & $0.0506(10)$ & $0.0033(8)$ & $-0.0043(8)$ & $-0.0084(8)$ \\
\hline N10 & $0.0471(11)$ & 0.0408 (10) & $0.0444(9)$ & $-0.0029(8)$ & $-0.0030(8)$ & $-0.0029(8)$ \\
\hline C11 & $0.0451(13)$ & $0.0420(11)$ & $0.0461(11)$ & $0.0020(9)$ & $0.0006(10)$ & $0.0014(9)$ \\
\hline C12 & 0.0458 (13) & $0.0404(11)$ & $0.0424(11)$ & $-0.0007(9)$ & $-0.0002(9)$ & $0.0024(9)$ \\
\hline C13 & $0.0611(16)$ & 0.0527 (14) & $0.0520(13)$ & $0.0196(11)$ & $-0.0082(11)$ & $-0.0087(10)$ \\
\hline C14 & $0.0600(15)$ & $0.0569(14)$ & $0.0515(13)$ & $0.0146(11)$ & $-0.0136(11)$ & $-0.0038(11)$ \\
\hline $\mathrm{C} 15$ & $0.0553(14)$ & $0.0429(12)$ & $0.0405(11)$ & $0.0003(10)$ & $-0.0008(10)$ & 0.0007 (9) \\
\hline C16 & $0.0616(16)$ & $0.0536(13)$ & 0.0535 (13) & 0.0137 (11) & $-0.0009(12)$ & $-0.0088(11)$ \\
\hline $\mathrm{C} 17$ & $0.0516(15)$ & $0.0545(13)$ & $0.0553(13)$ & $0.0117(11)$ & $-0.0082(11)$ & $-0.0018(11)$ \\
\hline $\mathrm{O} 18$ & 0.0748 (13) & $0.0574(10)$ & $0.0539(9)$ & $0.0122(8)$ & $-0.0116(8)$ & $-0.0129(8)$ \\
\hline
\end{tabular}

Geometric parameters $(\AA, \stackrel{o}{)})$

\begin{tabular}{llll}
\hline $\mathrm{S} 1 \mathrm{~A}-\mathrm{C} 2 \mathrm{~A}$ & $1.691(4)$ & $\mathrm{C} 7-\mathrm{O} 8$ & $1.240(2)$ \\
$\mathrm{C} 2 \mathrm{~A}-\mathrm{H} 2 \mathrm{~A}$ & 0.9300 & $\mathrm{C} 7-\mathrm{N} 9$ & $1.339(2)$ \\
$\mathrm{S} 1 \mathrm{~B}-\mathrm{C} 2 \mathrm{~B}$ & $1.677(9)$ & $\mathrm{N} 9-\mathrm{H} 9$ & 0.8600 \\
$\mathrm{C} 2 \mathrm{~B}-\mathrm{H} 2 \mathrm{~B}$ & 0.9300 & $\mathrm{~N} 9-\mathrm{N} 10$ & $1.391(2)$ \\
$\mathrm{C} 2 \mathrm{~A}-\mathrm{C} 3$ & $1.357(4)$ & $\mathrm{N} 10-\mathrm{C} 11$ & $1.277(2)$ \\
$\mathrm{C} 2 \mathrm{~B}-\mathrm{C} 3$ & $1.360(9)$ & $\mathrm{C} 11-\mathrm{H} 11$ & 0.9300 \\
$\mathrm{C} 4 \mathrm{~A}-\mathrm{H} 4 \mathrm{~A}$ & 0.9300 & $\mathrm{C} 11-\mathrm{C} 12$ & $1.461(3)$ \\
$\mathrm{C} 4 \mathrm{~B}-\mathrm{H} 4 \mathrm{~B}$ & 0.9300 & $\mathrm{C} 12-\mathrm{C} 13$ & $1.382(3)$ \\
$\mathrm{S} 1 \mathrm{~A}-\mathrm{C} 5 \mathrm{~A}$ & $1.700(7)$ & $\mathrm{C} 12-\mathrm{C} 17$ & $1.393(3)$ \\
$\mathrm{C} 4 \mathrm{~A}-\mathrm{C} 5 \mathrm{~A}$ & $1.404(6)$ & $\mathrm{C} 13-\mathrm{H} 13$ & 0.9300 \\
$\mathrm{C} 5 \mathrm{~A}-\mathrm{H} 5 \mathrm{~A}$ & 0.9300 & $\mathrm{C} 13-\mathrm{C} 14$ & $1.386(3)$ \\
$\mathrm{S} 1 \mathrm{~B}-\mathrm{C} 5 \mathrm{~B}$ & $1.688(9)$ & $\mathrm{C} 14-\mathrm{H} 14$ & 0.9300 \\
$\mathrm{C} 4 \mathrm{~B}-\mathrm{C} 5 \mathrm{~B}$ & $1.419(9)$ & $\mathrm{C} 14-\mathrm{C} 15$ & $1.381(3)$ \\
$\mathrm{C} 5 \mathrm{~B}-\mathrm{H} 5 \mathrm{~B}$ & 0.9300 & $\mathrm{C} 15-\mathrm{C} 16$ & $1.381(3)$ \\
$\mathrm{C} 3-\mathrm{C} 4 \mathrm{~A}$ & $1.407(4)$ & $\mathrm{C} 15-\mathrm{O} 18$ & $1.364(2)$ \\
$\mathrm{C} 3-\mathrm{C} 4 \mathrm{~B}$ & $1.404(9)$ & $\mathrm{C} 16-\mathrm{H} 16$ & 0.9300 \\
$\mathrm{C} 3-\mathrm{C} 6$ & $1.512(3)$ & $\mathrm{C} 16-\mathrm{C} 17$ & $1.376(3)$ \\
$\mathrm{C} 6-\mathrm{H} 6 \mathrm{~A}$ & 0.9700 & $\mathrm{C} 17-\mathrm{H} 17$ & 0.9300
\end{tabular}




\begin{tabular}{|c|c|c|c|}
\hline C6-H6B & 0.9700 & $\mathrm{O} 18-\mathrm{H} 18$ & 0.8200 \\
\hline $\mathrm{C} 6-\mathrm{C} 7$ & $1.508(3)$ & & \\
\hline $\mathrm{C} 4 \mathrm{~A}-\mathrm{C} 5 \mathrm{~A}-\mathrm{S} 1 \mathrm{~A}$ & $107.6(5)$ & $\mathrm{C} 3-\mathrm{C} 4 \mathrm{~B}-\mathrm{C} 5 \mathrm{~B}$ & $114.2(9)$ \\
\hline $\mathrm{C} 4 \mathrm{~B}-\mathrm{C} 5 \mathrm{~B}-\mathrm{S} 1 \mathrm{~B}$ & $107.2(8)$ & $\mathrm{O} 8-\mathrm{C} 7-\mathrm{C} 6$ & $121.54(19)$ \\
\hline $\mathrm{S} 1 \mathrm{~A}-\mathrm{C} 2 \mathrm{~A}-\mathrm{H} 2 \mathrm{~A}$ & 124.0 & $\mathrm{O} 8-\mathrm{C} 7-\mathrm{N} 9$ & $122.07(18)$ \\
\hline $\mathrm{S} 1 \mathrm{~B}-\mathrm{C} 2 \mathrm{~B}-\mathrm{H} 2 \mathrm{~B}$ & 124.2 & $\mathrm{~N} 9-\mathrm{C} 7-\mathrm{C} 6$ & $116.27(18)$ \\
\hline $\mathrm{C} 5 \mathrm{~A}-\mathrm{C} 4 \mathrm{~A}-\mathrm{C} 3$ & $115.0(5)$ & $\mathrm{C} 7-\mathrm{N} 9-\mathrm{H} 9$ & 120.4 \\
\hline $\mathrm{C} 5 \mathrm{~A}-\mathrm{C} 4 \mathrm{~A}-\mathrm{H} 4 \mathrm{~A}$ & 122.5 & $\mathrm{C} 7-\mathrm{N} 9-\mathrm{N} 10$ & $119.29(16)$ \\
\hline $\mathrm{C} 5 \mathrm{~B}-\mathrm{C} 4 \mathrm{~B}-\mathrm{H} 4 \mathrm{~B}$ & 122.9 & $\mathrm{~N} 10-\mathrm{N} 9-\mathrm{H} 9$ & 120.4 \\
\hline $\mathrm{C} 2 \mathrm{~A}-\mathrm{S} 1 \mathrm{~A}-\mathrm{C} 5 \mathrm{~A}$ & $94.3(3)$ & $\mathrm{C} 11-\mathrm{N} 10-\mathrm{N} 9$ & $115.25(17)$ \\
\hline $\mathrm{S} 1 \mathrm{~A}-\mathrm{C} 5 \mathrm{~A}-\mathrm{H} 5 \mathrm{~A}$ & 126.2 & $\mathrm{~N} 10-\mathrm{C} 11-\mathrm{H} 11$ & 119.1 \\
\hline $\mathrm{C} 4 \mathrm{~A}-\mathrm{C} 5 \mathrm{~A}-\mathrm{H} 5 \mathrm{~A}$ & 126.2 & $\mathrm{~N} 10-\mathrm{C} 11-\mathrm{C} 12$ & $121.81(19)$ \\
\hline $\mathrm{C} 2 \mathrm{~B}-\mathrm{S} 1 \mathrm{~B}-\mathrm{C} 5 \mathrm{~B}$ & $95.2(6)$ & $\mathrm{C} 12-\mathrm{C} 11-\mathrm{H} 11$ & 119.1 \\
\hline $\mathrm{S} 1 \mathrm{~B}-\mathrm{C} 5 \mathrm{~B}-\mathrm{H} 5 \mathrm{~B}$ & 126.4 & $\mathrm{C} 13-\mathrm{C} 12-\mathrm{C} 11$ & $120.45(18)$ \\
\hline $\mathrm{C} 4 \mathrm{~B}-\mathrm{C} 5 \mathrm{~B}-\mathrm{H} 5 \mathrm{~B}$ & 126.4 & $\mathrm{C} 13-\mathrm{C} 12-\mathrm{C} 17$ & $117.57(19)$ \\
\hline $\mathrm{C} 2 \mathrm{~A}-\mathrm{C} 3-\mathrm{C} 4 \mathrm{~A}$ & $111.1(3)$ & $\mathrm{C} 17-\mathrm{C} 12-\mathrm{C} 11$ & $121.95(19)$ \\
\hline $\mathrm{C} 2 \mathrm{~B}-\mathrm{C} 3-\mathrm{C} 4 \mathrm{~B}$ & $111.5(7)$ & $\mathrm{C} 12-\mathrm{C} 13-\mathrm{H} 13$ & 119.1 \\
\hline $\mathrm{C} 4 \mathrm{~A}-\mathrm{C} 3-\mathrm{C} 6$ & $125.0(3)$ & $\mathrm{C} 12-\mathrm{C} 13-\mathrm{C} 14$ & $121.7(2)$ \\
\hline $\mathrm{C} 2 \mathrm{~A}-\mathrm{C} 3-\mathrm{C} 6$ & $123.9(3)$ & $\mathrm{C} 14-\mathrm{C} 13-\mathrm{H} 13$ & 119.1 \\
\hline $\mathrm{C} 4 \mathrm{~B}-\mathrm{C} 3-\mathrm{C} 6$ & $128.0(6)$ & $\mathrm{C} 13-\mathrm{C} 14-\mathrm{H} 14$ & 120.2 \\
\hline $\mathrm{C} 2 \mathrm{~B}-\mathrm{C} 3-\mathrm{C} 6$ & $120.4(5)$ & $\mathrm{C} 15-\mathrm{C} 14-\mathrm{C} 13$ & $119.6(2)$ \\
\hline $\mathrm{C} 3-\mathrm{C} 6-\mathrm{H} 6 \mathrm{~A}$ & 110.0 & $\mathrm{C} 15-\mathrm{C} 14-\mathrm{H} 14$ & 120.2 \\
\hline $\mathrm{C} 3-\mathrm{C} 2 \mathrm{~A}-\mathrm{S} 1 \mathrm{~A}$ & $112.1(3)$ & $\mathrm{C} 14-\mathrm{C} 15-\mathrm{C} 16$ & $119.6(2)$ \\
\hline $\mathrm{C} 3-\mathrm{C} 2 \mathrm{~B}-\mathrm{S} 1 \mathrm{~B}$ & $111.6(7)$ & $\mathrm{O} 18-\mathrm{C} 15-\mathrm{C} 14$ & $123.0(2)$ \\
\hline $\mathrm{C} 3-\mathrm{C} 6-\mathrm{H} 6 \mathrm{~B}$ & 110.0 & $\mathrm{O} 18-\mathrm{C} 15-\mathrm{C} 16$ & $117.47(19)$ \\
\hline $\mathrm{H} 6 \mathrm{~A}-\mathrm{C} 6-\mathrm{H} 6 \mathrm{~B}$ & 108.3 & $\mathrm{C} 15-\mathrm{C} 16-\mathrm{H} 16$ & 119.9 \\
\hline $\mathrm{C} 3-\mathrm{C} 2 \mathrm{~A}-\mathrm{H} 2 \mathrm{~A}$ & 124.0 & $\mathrm{C} 17-\mathrm{C} 16-\mathrm{C} 15$ & $120.3(2)$ \\
\hline $\mathrm{C} 3-\mathrm{C} 2 \mathrm{~B}-\mathrm{H} 2 \mathrm{~B}$ & 124.2 & $\mathrm{C} 17-\mathrm{C} 16-\mathrm{H} 16$ & 119.9 \\
\hline $\mathrm{C} 7-\mathrm{C} 6-\mathrm{C} 3$ & $108.68(16)$ & $\mathrm{C} 12-\mathrm{C} 17-\mathrm{H} 17$ & 119.4 \\
\hline $\mathrm{C} 7-\mathrm{C} 6-\mathrm{H} 6 \mathrm{~A}$ & 110.0 & $\mathrm{C} 16-\mathrm{C} 17-\mathrm{C} 12$ & $121.3(2)$ \\
\hline $\mathrm{C} 3-\mathrm{C} 4 \mathrm{~A}-\mathrm{H} 4 \mathrm{~A}$ & 122.5 & $\mathrm{C} 16-\mathrm{C} 17-\mathrm{H} 17$ & 119.4 \\
\hline $\mathrm{C} 3-\mathrm{C} 4 \mathrm{~B}-\mathrm{H} 4 \mathrm{~B}$ & 122.9 & $\mathrm{C} 15-\mathrm{O} 18-\mathrm{H} 18$ & 109.5 \\
\hline $\mathrm{C} 7-\mathrm{C} 6-\mathrm{H} 6 \mathrm{~B}$ & 110.0 & & \\
\hline $\mathrm{C} 2 \mathrm{~A}-\mathrm{S} 1 \mathrm{~A}-\mathrm{C} 5 \mathrm{~A}-\mathrm{C} 4 \mathrm{~A}$ & $-0.7(11)$ & $\mathrm{C} 6-\mathrm{C} 3-\mathrm{C} 4 \mathrm{~B}-\mathrm{C} 5 \mathrm{~B}$ & $176.8(17)$ \\
\hline $\mathrm{C} 2 \mathrm{~B}-\mathrm{S} 1 \mathrm{~B}-\mathrm{C} 5 \mathrm{~B}-\mathrm{C} 4 \mathrm{~B}$ & $5(3)$ & $\mathrm{C} 6-\mathrm{C} 3-\mathrm{C} 4 \mathrm{~A}-\mathrm{C} 5 \mathrm{~A}$ & $-177.0(8)$ \\
\hline $\mathrm{C} 5 \mathrm{~B}-\mathrm{S} 1 \mathrm{~B}-\mathrm{C} 2 \mathrm{~B}-\mathrm{C} 3$ & $-5(3)$ & $\mathrm{C} 6-\mathrm{C} 7-\mathrm{N} 9-\mathrm{N} 10$ & $167.45(16)$ \\
\hline $\mathrm{C} 5 \mathrm{~A}-\mathrm{S} 1 \mathrm{~A}-\mathrm{C} 2 \mathrm{~A}-\mathrm{C} 3$ & $0.8(7)$ & $\mathrm{C} 7-\mathrm{N} 9-\mathrm{N} 10-\mathrm{C} 11$ & $177.10(18)$ \\
\hline $\mathrm{S} 1 \mathrm{~B}-\mathrm{C} 2 \mathrm{~B}-\mathrm{C} 3-\mathrm{C} 4 \mathrm{~B}$ & $3(3)$ & $\mathrm{O} 8-\mathrm{C} 7-\mathrm{N} 9-\mathrm{N} 10$ & $-8.6(3)$ \\
\hline $\mathrm{S} 1 \mathrm{~A}-\mathrm{C} 2 \mathrm{~A}-\mathrm{C} 3-\mathrm{C} 4 \mathrm{~A}$ & $-0.7(5)$ & $\mathrm{N} 9-\mathrm{N} 10-\mathrm{C} 11-\mathrm{C} 12$ & $174.82(16)$ \\
\hline $\mathrm{S} 1 \mathrm{~A}-\mathrm{C} 2 \mathrm{~A}-\mathrm{C} 3-\mathrm{C} 6$ & $176.5(3)$ & $\mathrm{N} 10-\mathrm{C} 11-\mathrm{C} 12-\mathrm{C} 13$ & $169.1(2)$ \\
\hline $\mathrm{S} 1 \mathrm{~B}-\mathrm{C} 2 \mathrm{~B}-\mathrm{C} 3-\mathrm{C} 6$ & $-173.1(14)$ & $\mathrm{N} 10-\mathrm{C} 11-\mathrm{C} 12-\mathrm{C} 17$ & $-12.8(3)$ \\
\hline $\mathrm{C} 2 \mathrm{~B}-\mathrm{C} 3-\mathrm{C} 6-\mathrm{C} 7$ & $95(2)$ & $\mathrm{C} 11-\mathrm{C} 12-\mathrm{C} 13-\mathrm{C} 14$ & $179.8(2)$ \\
\hline $\mathrm{C} 2 \mathrm{~A}-\mathrm{C} 3-\mathrm{C} 6-\mathrm{C} 7$ & $-91.1(5)$ & $\mathrm{C} 11-\mathrm{C} 12-\mathrm{C} 17-\mathrm{C} 16$ & $-179.4(2)$ \\
\hline $\mathrm{C} 4 \mathrm{~A}-\mathrm{C} 3-\mathrm{C} 6-\mathrm{C} 7$ & $85.7(7)$ & $\mathrm{C} 12-\mathrm{C} 13-\mathrm{C} 14-\mathrm{C} 15$ & $-0.9(4)$ \\
\hline $\mathrm{C} 4 \mathrm{~B}-\mathrm{C} 3-\mathrm{C} 6-\mathrm{C} 7$ & $-81.0(17)$ & $\mathrm{C} 13-\mathrm{C} 12-\mathrm{C} 17-\mathrm{C} 16$ & $-1.2(3)$ \\
\hline $\mathrm{C} 2 \mathrm{~A}-\mathrm{C} 3-\mathrm{C} 4 \mathrm{~A}-\mathrm{C} 5 \mathrm{~A}$ & $0.1(11)$ & $\mathrm{C} 13-\mathrm{C} 14-\mathrm{C} 15-\mathrm{C} 16$ & $-0.1(3)$ \\
\hline
\end{tabular}




$\begin{array}{llll}\mathrm{C} 2 \mathrm{~B}-\mathrm{C} 3-\mathrm{C} 4 \mathrm{~B}-\mathrm{C} 5 \mathrm{~B} & 1(3) & \mathrm{C} 13-\mathrm{C} 14-\mathrm{C} 15-\mathrm{O} 18 & 179.4(2) \\ \mathrm{C} 3-\mathrm{C} 4 \mathrm{~A}-\mathrm{C} 5 \mathrm{~A}-\mathrm{S} 1 \mathrm{~A} & 0.5(14) & \mathrm{C} 14-\mathrm{C} 15-\mathrm{C} 16-\mathrm{C} 17 & 0.5(3) \\ \mathrm{C} 3-\mathrm{C} 4 \mathrm{~B}-\mathrm{C} 5 \mathrm{~B}-\mathrm{S} 1 \mathrm{~B} & -4(3) & \mathrm{C} 15-\mathrm{C} 16-\mathrm{C} 17-\mathrm{C} 12 & 0.1(3) \\ \mathrm{C} 3-\mathrm{C} 6-\mathrm{C} 7-\mathrm{O} 8 & 74.2(2) & \mathrm{C} 17-\mathrm{C} 12-\mathrm{C} 13-\mathrm{C} 14 & 1.6(3) \\ \mathrm{C} 3-\mathrm{C} 6-\mathrm{C} 7-\mathrm{N} 9 & -101.8(2) & \mathrm{O} 18-\mathrm{C} 15-\mathrm{C} 16-\mathrm{C} 17 & -179.0(2)\end{array}$

Hydrogen-bond geometry $\left(\AA,{ }^{\circ}\right)$

$\mathrm{Cg} 3$ is the centroid of the $\mathrm{C} 12-\mathrm{C} 17$ phenyl ring.

\begin{tabular}{lllll}
\hline$D-\mathrm{H} \cdots A$ & $D-\mathrm{H}$ & $\mathrm{H} \cdots A$ & $D \cdots A$ & $D-\mathrm{H} \cdots A$ \\
\hline $\mathrm{N} 9-\mathrm{H} 9 \cdots \mathrm{O}^{\mathrm{i}}$ & 0.86 & 2.12 & $2.953(2)$ & 162 \\
$\mathrm{O} 18-\mathrm{H} 18 \cdots 8^{\mathrm{ii}}$ & 0.82 & 1.97 & $2.782(2)$ & 169 \\
$\mathrm{C} 2 A-\mathrm{H} 2 A \cdots \mathrm{O} 8^{\mathrm{iii}}$ & 0.93 & 2.57 & $3.439(7)$ & 155 \\
$\mathrm{C} 13-\mathrm{H} 13 \cdots \mathrm{Cg}^{\mathrm{iiv}}$ & 0.93 & 2.89 & $3.818(3)$ & 176
\end{tabular}

Symmetry codes: (i) $-x+1 / 2, y+1 / 2, z$; (ii) $x-1 / 2, y,-z+1 / 2$; (iii) $-x+1,-y+1,-z+1$; (iv) $-x, y+1 / 2,-z+1 / 2$.

$N^{\prime}$-[1-(4-Methoxyphenyl)benzylidene]-2-(thiophen-3-yl)acetohydrazide (3b)

\section{Crystal data}

$\mathrm{C}_{14} \mathrm{H}_{14} \mathrm{~N}_{2} \mathrm{O}_{2} \mathrm{~S}$

$M_{r}=274.33$

Triclinic, $P \overline{1}$

$a=6.5185(2) \AA$

$b=9.7447(5) \AA$

$c=10.9291(6) \AA$

$\alpha=78.327(4)^{\circ}$

$\beta=83.070(4)^{\circ}$

$\gamma=87.013(4)^{\circ}$

$V=674.63(6) \AA^{3}$

\section{Data collection}

Rigaku Oxford Diffraction SuperNova, Single source at offset/far, Eos diffractometer

Radiation source: micro-focus sealed X-ray tube, SuperNova (Mo) X-ray Source

Mirror monochromator

Detector resolution: 15.9631 pixels $\mathrm{mm}^{-1}$

$\omega$ scans

Absorption correction: multi-scan

(CrysAlis PRO; Rigaku OD, 2018)

\section{Refinement}

Refinement on $F^{2}$

Least-squares matrix: full

$R\left[F^{2}>2 \sigma\left(F^{2}\right)\right]=0.051$

$w R\left(F^{2}\right)=0.145$

$S=1.06$

2752 reflections

173 parameters

0 restraints
$Z=2$

$F(000)=288$

$D_{\mathrm{x}}=1.350 \mathrm{Mg} \mathrm{m}^{-3}$

Mo $K \alpha$ radiation, $\lambda=0.71073 \AA$

Cell parameters from 5534 reflections

$\theta=3.1-27.2^{\circ}$

$\mu=0.24 \mathrm{~mm}^{-1}$

$T=293 \mathrm{~K}$

Needle, white

$0.5 \times 0.15 \times 0.05 \mathrm{~mm}$

$T_{\min }=0.687, T_{\max }=1.000$

13795 measured reflections

2752 independent reflections

2238 reflections with $I>2 \sigma(I)$

$R_{\text {int }}=0.027$

$\theta_{\text {max }}=26.4^{\circ}, \theta_{\min }=2.6^{\circ}$

$h=-8 \rightarrow 8$

$k=-12 \rightarrow 12$

$l=-13 \rightarrow 13$

Hydrogen site location: inferred from neighbouring sites

$\mathrm{H}$-atom parameters constrained

$w=1 /\left[\sigma^{2}\left(F_{\mathrm{o}}^{2}\right)+(0.0537 P)^{2}+0.5294 P\right]$

where $P=\left(F_{\mathrm{o}}{ }^{2}+2 F_{\mathrm{c}}{ }^{2}\right) / 3$

$(\Delta / \sigma)_{\max }<0.001$

$\Delta \rho_{\max }=0.33$ e $\AA^{-3}$

$\Delta \rho_{\min }=-0.38$ e $\AA^{-3}$ 


\section{Special details}

Geometry. All esds (except the esd in the dihedral angle between two 1.s. planes) are estimated using the full covariance matrix. The cell esds are taken into account individually in the estimation of esds in distances, angles and torsion angles; correlations between esds in cell parameters are only used when they are defined by crystal symmetry. An approximate (isotropic) treatment of cell esds is used for estimating esds involving l.s. planes.

Fractional atomic coordinates and isotropic or equivalent isotropic displacement parameters $\left(\hat{A}^{2}\right)$

\begin{tabular}{|c|c|c|c|c|}
\hline & $x$ & $y$ & $z$ & $U_{\text {iso }} * / U_{\text {eq }}$ \\
\hline S1 & $0.68895(12)$ & $0.78924(10)$ & $0.16957(9)$ & $0.0734(3)$ \\
\hline $\mathrm{C} 2$ & $0.5595(4)$ & $0.7356(3)$ & $0.3147(3)$ & $0.0534(6)$ \\
\hline $\mathrm{H} 2$ & 0.619153 & 0.726965 & 0.389168 & $0.064 *$ \\
\hline $\mathrm{C} 3$ & $0.3579(4)$ & $0.7066(2)$ & $0.3100(2)$ & $0.0436(5)$ \\
\hline $\mathrm{C} 4$ & $0.3123(4)$ & $0.7275(3)$ & $0.1841(2)$ & $0.0512(6)$ \\
\hline $\mathrm{H} 4$ & 0.182310 & 0.712513 & 0.163071 & $0.061^{*}$ \\
\hline $\mathrm{C} 5$ & $0.4790(4)$ & $0.7727(3)$ & $0.0935(2)$ & $0.0497(6)$ \\
\hline H5 & 0.476097 & 0.790729 & 0.006852 & $0.060 *$ \\
\hline C6 & $0.2002(4)$ & $0.6708(3)$ & $0.4234(2)$ & $0.0479(6)$ \\
\hline H6A & 0.265853 & 0.615910 & 0.493142 & $0.057^{*}$ \\
\hline H6B & 0.093072 & 0.615347 & 0.404620 & $0.057^{*}$ \\
\hline $\mathrm{C} 7$ & $0.1056(3)$ & $0.8042(3)$ & $0.4589(2)$ & $0.0418(5)$ \\
\hline O8 & $-0.0513(2)$ & $0.86009(19)$ & $0.41630(16)$ & $0.0508(4)$ \\
\hline N9 & $0.2051(3)$ & $0.8658(2)$ & $0.53376(18)$ & $0.0417(5)$ \\
\hline H9 & 0.160089 & 0.945717 & 0.549350 & $0.050 *$ \\
\hline N10 & $0.3778(3)$ & $0.8031(2)$ & $0.58594(17)$ & $0.0412(5)$ \\
\hline C11 & $0.4621(3)$ & $0.8739(2)$ & $0.6517(2)$ & $0.0411(5)$ \\
\hline H11 & 0.406000 & 0.961642 & 0.660165 & $0.049 *$ \\
\hline $\mathrm{C} 12$ & $0.6438(3)$ & $0.8207(2)$ & $0.7139(2)$ & $0.0372(5)$ \\
\hline $\mathrm{C} 13$ & $0.7354(4)$ & $0.9057(2)$ & $0.7794(2)$ & $0.0423(5)$ \\
\hline H13 & 0.679005 & 0.994741 & 0.782354 & $0.051 *$ \\
\hline C14 & $0.9070(4)$ & $0.8605(2)$ & $0.8396(2)$ & $0.0444(5)$ \\
\hline H14 & 0.966061 & 0.919004 & 0.882266 & $0.053^{*}$ \\
\hline $\mathrm{C} 15$ & $0.9921(3)$ & $0.7276(2)$ & $0.8368(2)$ & $0.0409(5)$ \\
\hline $\mathrm{C} 16$ & 0.9037 (4) & $0.6415(2)$ & $0.7722(2)$ & $0.0442(5)$ \\
\hline H16 & 0.960075 & 0.552276 & 0.769944 & $0.053^{*}$ \\
\hline $\mathrm{C} 17$ & $0.7322(4)$ & $0.6880(2)$ & $0.7113(2)$ & $0.0435(5)$ \\
\hline H17 & 0.674636 & 0.629647 & 0.667808 & $0.052 *$ \\
\hline $\mathrm{O} 18$ & $1.1602(3)$ & 0.69079 (19) & $0.90073(17)$ & $0.0565(5)$ \\
\hline C19 & $1.2603(4)$ & $0.5592(3)$ & $0.8926(3)$ & $0.0634(8)$ \\
\hline H19A & 1.307244 & 0.557208 & 0.806228 & $0.095^{*}$ \\
\hline H19B & 1.164788 & 0.485433 & 0.925510 & $0.095^{*}$ \\
\hline $\mathrm{H} 19 \mathrm{C}$ & 1.376398 & 0.546115 & 0.940466 & $0.095^{*}$ \\
\hline
\end{tabular}

Atomic displacement parameters $\left(\AA^{2}\right)$

\begin{tabular}{lllllll}
\hline & $U^{11}$ & $U^{22}$ & $U^{33}$ & $U^{12}$ & $U^{13}$ & $U^{23}$ \\
\hline S1 & $0.0554(5)$ & $0.0848(6)$ & $0.0861(6)$ & $-0.0076(4)$ & $0.0084(4)$ & $-0.0394(5)$ \\
C2 & $0.0441(13)$ & $0.0592(16)$ & $0.0640(16)$ & $0.0019(11)$ & $-0.0103(12)$ & $-0.0270(13)$
\end{tabular}




\begin{tabular}{lllllll} 
C3 & $0.0445(13)$ & $0.0376(12)$ & $0.0529(14)$ & $-0.0006(9)$ & $-0.0085(10)$ & $-0.0175(10)$ \\
C4 & $0.0541(15)$ & $0.0493(14)$ & $0.0568(15)$ & $-0.0023(11)$ & $-0.0156(12)$ & $-0.0207(12)$ \\
C5 & $0.0528(14)$ & $0.0505(14)$ & $0.0506(14)$ & $-0.0044(11)$ & $0.0003(11)$ & $-0.0238(11)$ \\
C6 & $0.0503(14)$ & $0.0436(13)$ & $0.0526(14)$ & $-0.0098(10)$ & $-0.0110(11)$ & $-0.0108(11)$ \\
C7 & $0.0368(12)$ & $0.0511(13)$ & $0.0368(11)$ & $-0.0088(10)$ & $-0.0037(9)$ & $-0.0056(10)$ \\
O8 & $0.0366(9)$ & $0.0680(12)$ & $0.0511(10)$ & $-0.0008(8)$ & $-0.0129(7)$ & $-0.0152(8)$ \\
N9 & $0.0364(10)$ & $0.0489(11)$ & $0.0424(10)$ & $0.0032(8)$ & $-0.0114(8)$ & $-0.0121(8)$ \\
N10 & $0.0381(10)$ & $0.0472(11)$ & $0.0386(10)$ & $0.0011(8)$ & $-0.0096(8)$ & $-0.0069(8)$ \\
C11 & $0.0421(12)$ & $0.0422(12)$ & $0.0396(12)$ & $0.0021(9)$ & $-0.0073(9)$ & $-0.0087(9)$ \\
C12 & $0.0370(11)$ & $0.0399(12)$ & $0.0343(11)$ & $-0.0020(9)$ & $-0.0055(9)$ & $-0.0052(9)$ \\
C13 & $0.0485(13)$ & $0.0361(12)$ & $0.0436(12)$ & $0.0034(10)$ & $-0.0101(10)$ & $-0.0098(9)$ \\
C14 & $0.0498(13)$ & $0.0439(13)$ & $0.0444(13)$ & $-0.0029(10)$ & $-0.0149(10)$ & $-0.0143(10)$ \\
C15 & $0.0393(12)$ & $0.0467(13)$ & $0.0356(11)$ & $-0.0006(10)$ & $-0.0088(9)$ & $-0.0034(9)$ \\
C16 & $0.0482(13)$ & $0.0365(12)$ & $0.0491(13)$ & $0.0036(10)$ & $-0.0117(11)$ & $-0.0094(10)$ \\
C17 & $0.0470(13)$ & $0.0408(12)$ & $0.0465(13)$ & $-0.0032(10)$ & $-0.0113(10)$ & $-0.0135(10)$ \\
O18 & $0.0538(10)$ & $0.0595(11)$ & $0.0613(11)$ & $0.0109(8)$ & $-0.0284(9)$ & $-0.0142(9)$ \\
C19 & $0.0560(16)$ & $0.0645(18)$ & $0.0690(18)$ & $0.0175(13)$ & $-0.0214(14)$ & $-0.0085(14)$ \\
& & & & & & \\
\hline
\end{tabular}

Geometric parameters $\left(A,{ }^{\circ}\right)$

\begin{tabular}{llll}
\hline $\mathrm{S} 1-\mathrm{C} 2$ & $1.700(3)$ & $\mathrm{C} 11-\mathrm{H} 11$ & 0.9300 \\
$\mathrm{~S} 1-\mathrm{C} 5$ & $1.715(3)$ & $\mathrm{C} 11-\mathrm{C} 12$ & $1.456(3)$ \\
$\mathrm{C} 2-\mathrm{H} 2$ & 0.9300 & $\mathrm{C} 12-\mathrm{C} 13$ & $1.396(3)$ \\
$\mathrm{C} 2-\mathrm{C} 3$ & $1.368(3)$ & $\mathrm{C} 12-\mathrm{C} 17$ & $1.393(3)$ \\
$\mathrm{C} 3-\mathrm{C} 4$ & $1.415(3)$ & $\mathrm{C} 13-\mathrm{H} 13$ & 0.9300 \\
$\mathrm{C} 3-\mathrm{C} 6$ & $1.507(3)$ & $\mathrm{C} 13-\mathrm{C} 14$ & 0.9300 \\
$\mathrm{C} 4-\mathrm{H} 4$ & 0.9300 & $\mathrm{C} 14-\mathrm{H} 14$ & $1.387(3)$ \\
$\mathrm{C} 4-\mathrm{C} 5$ & $1.403(4)$ & $\mathrm{C} 14-\mathrm{C} 15$ & $1.387(3)$ \\
$\mathrm{C} 5-\mathrm{H} 5$ & 0.9300 & $\mathrm{C} 15-\mathrm{C} 16$ & $1.363(3)$ \\
$\mathrm{C} 6-\mathrm{H} 6 \mathrm{~A}$ & 0.9700 & $\mathrm{C} 15-\mathrm{O} 18$ & 0.9300 \\
$\mathrm{C} 6-\mathrm{H} 6 \mathrm{~B}$ & 0.9700 & $\mathrm{C} 16-\mathrm{H} 16$ & $1.379(3)$ \\
$\mathrm{C} 6-\mathrm{C} 7$ & $1.511(3)$ & $\mathrm{C} 16-\mathrm{C} 17$ & 0.9300 \\
$\mathrm{C} 7-\mathrm{O} 8$ & $1.230(3)$ & $\mathrm{C} 17-\mathrm{H} 17$ & $1.422(3)$ \\
$\mathrm{C} 7-\mathrm{N} 9$ & $1.348(3)$ & $\mathrm{O} 18-\mathrm{C} 19$ & 0.9600 \\
$\mathrm{~N} 9-\mathrm{H} 9$ & 0.8600 & $\mathrm{C} 19-\mathrm{H} 19 \mathrm{~A}$ & 0.9600 \\
$\mathrm{~N} 9-\mathrm{N} 10$ & $1.382(2)$ & $\mathrm{C} 19-\mathrm{H} 19 \mathrm{~B}$ & \\
$\mathrm{~N} 10-\mathrm{C} 11$ & $1.277(3)$ & $\mathrm{C} 19-\mathrm{H} 19 \mathrm{C}$ & $121.7(2)$ \\
$\mathrm{C} 2-\mathrm{S} 1-\mathrm{C} 5$ & & & 119.2 \\
$\mathrm{~S} 1-\mathrm{C} 2-\mathrm{H} 2$ & $93.48(13)$ & $\mathrm{N} 10-\mathrm{C} 11-\mathrm{C} 12$ & $119.1(2)$ \\
$\mathrm{C} 3-\mathrm{C} 2-\mathrm{S} 1$ & 123.7 & $\mathrm{C} 12-\mathrm{C} 11-\mathrm{H} 11$ & $123.1(2)$ \\
$\mathrm{C} 3-\mathrm{C} 2-\mathrm{H} 2$ & $112.5(2)$ & $\mathrm{C} 13-\mathrm{C} 12-\mathrm{C} 11$ & $117.8(2)$ \\
$\mathrm{C} 2-\mathrm{C} 3-\mathrm{C} 4$ & 123.7 & $\mathrm{C} 17-\mathrm{C} 12-\mathrm{C} 11$ & 119.3 \\
$\mathrm{C} 2-\mathrm{C} 3-\mathrm{C} 6$ & $111.0(2)$ & $\mathrm{C} 17-\mathrm{C} 12-\mathrm{C} 13$ & $121.4(2)$ \\
$\mathrm{C} 4-\mathrm{C} 3-\mathrm{C} 6$ & $124.5(2)$ & $\mathrm{C} 12-\mathrm{C} 13-\mathrm{H} 13$ & 119.3 \\
$\mathrm{C} 3-\mathrm{C} 4-\mathrm{H} 4$ & $124.3(2)$ & $\mathrm{C} 14-\mathrm{C} 13-\mathrm{C} 12$ & 120.0 \\
$\mathrm{C} 5-\mathrm{C} 4-\mathrm{C} 3$ & 122.7 & $\mathrm{C} 14-\mathrm{C} 13-\mathrm{H} 13$ & $120.0(2)$ \\
$\mathrm{C} 5-\mathrm{C} 4-\mathrm{H} 4$ & $114.5(2)$ & $\mathrm{C} 13-\mathrm{C} 14-\mathrm{H} 14$ & \\
& 122.7 & $\mathrm{C} 13-\mathrm{C} 14-\mathrm{C} 15$ & \\
& & &
\end{tabular}




\begin{tabular}{|c|c|c|c|}
\hline $\mathrm{S} 1-\mathrm{C} 5-\mathrm{H} 5$ & 125.7 & $\mathrm{C} 15-\mathrm{C} 14-\mathrm{H} 14$ & 120.0 \\
\hline $\mathrm{C} 4-\mathrm{C} 5-\mathrm{S} 1$ & $108.50(19)$ & $\mathrm{C} 14-\mathrm{C} 15-\mathrm{C} 16$ & $119.5(2)$ \\
\hline $\mathrm{C} 4-\mathrm{C} 5-\mathrm{H} 5$ & 125.7 & $\mathrm{O} 18-\mathrm{C} 15-\mathrm{C} 14$ & $116.0(2)$ \\
\hline $\mathrm{C} 3-\mathrm{C} 6-\mathrm{H} 6 \mathrm{~A}$ & 109.8 & $\mathrm{O} 18-\mathrm{C} 15-\mathrm{C} 16$ & $124.5(2)$ \\
\hline $\mathrm{C} 3-\mathrm{C} 6-\mathrm{H} 6 \mathrm{~B}$ & 109.8 & $\mathrm{C} 15-\mathrm{C} 16-\mathrm{H} 16$ & 119.9 \\
\hline $\mathrm{C} 3-\mathrm{C} 6-\mathrm{C} 7$ & $109.57(19)$ & $\mathrm{C} 17-\mathrm{C} 16-\mathrm{C} 15$ & $120.1(2)$ \\
\hline $\mathrm{H} 6 \mathrm{~A}-\mathrm{C} 6-\mathrm{H} 6 \mathrm{~B}$ & 108.2 & $\mathrm{C} 17-\mathrm{C} 16-\mathrm{H} 16$ & 119.9 \\
\hline C7-C6-H6A & 109.8 & $\mathrm{C} 12-\mathrm{C} 17-\mathrm{H} 17$ & 119.4 \\
\hline $\mathrm{C} 7-\mathrm{C} 6-\mathrm{H} 6 \mathrm{~B}$ & 109.8 & $\mathrm{C} 16-\mathrm{C} 17-\mathrm{C} 12$ & $121.2(2)$ \\
\hline $\mathrm{O} 8-\mathrm{C} 7-\mathrm{C} 6$ & $121.7(2)$ & $\mathrm{C} 16-\mathrm{C} 17-\mathrm{H} 17$ & 119.4 \\
\hline $\mathrm{O} 8-\mathrm{C} 7-\mathrm{N} 9$ & $120.2(2)$ & $\mathrm{C} 15-\mathrm{O} 18-\mathrm{C} 19$ & $117.5(2)$ \\
\hline $\mathrm{N} 9-\mathrm{C} 7-\mathrm{C} 6$ & $117.9(2)$ & $\mathrm{O} 18-\mathrm{C} 19-\mathrm{H} 19 \mathrm{~A}$ & 109.5 \\
\hline $\mathrm{C} 7-\mathrm{N} 9-\mathrm{H} 9$ & 119.3 & $\mathrm{O} 18-\mathrm{C} 19-\mathrm{H} 19 \mathrm{~B}$ & 109.5 \\
\hline $\mathrm{C} 7-\mathrm{N} 9-\mathrm{N} 10$ & $121.3(2)$ & $\mathrm{O} 18-\mathrm{C} 19-\mathrm{H} 19 \mathrm{C}$ & 109.5 \\
\hline N10-N9-H9 & 119.3 & $\mathrm{H} 19 \mathrm{~A}-\mathrm{C} 19-\mathrm{H} 19 \mathrm{~B}$ & 109.5 \\
\hline $\mathrm{C} 11-\mathrm{N} 10-\mathrm{N} 9$ & $115.4(2)$ & $\mathrm{H} 19 \mathrm{~A}-\mathrm{C} 19-\mathrm{H} 19 \mathrm{C}$ & 109.5 \\
\hline $\mathrm{N} 10-\mathrm{C} 11-\mathrm{H} 11$ & 119.2 & $\mathrm{H} 19 \mathrm{~B}-\mathrm{C} 19-\mathrm{H} 19 \mathrm{C}$ & 109.5 \\
\hline $\mathrm{S} 1-\mathrm{C} 2-\mathrm{C} 3-\mathrm{C} 4$ & $0.8(3)$ & $\mathrm{N} 10-\mathrm{C} 11-\mathrm{C} 12-\mathrm{C} 13$ & $176.9(2)$ \\
\hline $\mathrm{S} 1-\mathrm{C} 2-\mathrm{C} 3-\mathrm{C} 6$ & $-173.66(19)$ & $\mathrm{N} 10-\mathrm{C} 11-\mathrm{C} 12-\mathrm{C} 17$ & $-3.1(4)$ \\
\hline $\mathrm{C} 2-\mathrm{S} 1-\mathrm{C} 5-\mathrm{C} 4$ & $0.8(2)$ & $\mathrm{C} 11-\mathrm{C} 12-\mathrm{C} 13-\mathrm{C} 14$ & $180.0(2)$ \\
\hline $\mathrm{C} 2-\mathrm{C} 3-\mathrm{C} 4-\mathrm{C} 5$ & $-0.2(3)$ & $\mathrm{C} 11-\mathrm{C} 12-\mathrm{C} 17-\mathrm{C} 16$ & $-179.6(2)$ \\
\hline $\mathrm{C} 2-\mathrm{C} 3-\mathrm{C} 6-\mathrm{C} 7$ & $84.9(3)$ & $\mathrm{C} 12-\mathrm{C} 13-\mathrm{C} 14-\mathrm{C} 15$ & $-0.4(4)$ \\
\hline $\mathrm{C} 3-\mathrm{C} 4-\mathrm{C} 5-\mathrm{S} 1$ & $-0.5(3)$ & $\mathrm{C} 13-\mathrm{C} 12-\mathrm{C} 17-\mathrm{C} 16$ & $0.4(3)$ \\
\hline $\mathrm{C} 3-\mathrm{C} 6-\mathrm{C} 7-\mathrm{O} 8$ & $90.8(3)$ & $\mathrm{C} 13-\mathrm{C} 14-\mathrm{C} 15-\mathrm{C} 16$ & $0.4(4)$ \\
\hline $\mathrm{C} 3-\mathrm{C} 6-\mathrm{C} 7-\mathrm{N} 9$ & $-85.4(3)$ & $\mathrm{C} 13-\mathrm{C} 14-\mathrm{C} 15-\mathrm{O} 18$ & $-179.0(2)$ \\
\hline $\mathrm{C} 4-\mathrm{C} 3-\mathrm{C} 6-\mathrm{C} 7$ & $-88.8(3)$ & $\mathrm{C} 14-\mathrm{C} 15-\mathrm{C} 16-\mathrm{C} 17$ & $0.0(4)$ \\
\hline $\mathrm{C} 5-\mathrm{S} 1-\mathrm{C} 2-\mathrm{C} 3$ & $-1.0(2)$ & $\mathrm{C} 14-\mathrm{C} 15-\mathrm{O} 18-\mathrm{C} 19$ & $-175.7(2)$ \\
\hline $\mathrm{C} 6-\mathrm{C} 3-\mathrm{C} 4-\mathrm{C} 5$ & $174.3(2)$ & $\mathrm{C} 15-\mathrm{C} 16-\mathrm{C} 17-\mathrm{C} 12$ & $-0.4(4)$ \\
\hline $\mathrm{C} 6-\mathrm{C} 7-\mathrm{N} 9-\mathrm{N} 10$ & $-5.8(3)$ & $\mathrm{C} 16-\mathrm{C} 15-\mathrm{O} 18-\mathrm{C} 19$ & $4.9(4)$ \\
\hline $\mathrm{C} 7-\mathrm{N} 9-\mathrm{N} 10-\mathrm{C} 11$ & $177.8(2)$ & $\mathrm{C} 17-\mathrm{C} 12-\mathrm{C} 13-\mathrm{C} 14$ & $0.0(3)$ \\
\hline $\mathrm{O} 8-\mathrm{C} 7-\mathrm{N} 9-\mathrm{N} 10$ & $177.97(19)$ & $\mathrm{O} 18-\mathrm{C} 15-\mathrm{C} 16-\mathrm{C} 17$ & $179.4(2)$ \\
\hline $\mathrm{N} 9-\mathrm{N} 10-\mathrm{C} 11-\mathrm{C} 12$ & $179.26(19)$ & & \\
\hline
\end{tabular}

Hydrogen-bond geometry $\left(\AA,{ }^{\circ}\right)$

$C g 1$ is the centroid of the $\mathrm{S} 1 / \mathrm{C} 1-\mathrm{C} 5$ thiophene ring.

\begin{tabular}{lllll}
\hline$D-\mathrm{H} \cdots A$ & $D-\mathrm{H}$ & $\mathrm{H} \cdots A$ & $D \cdots A$ & $D-\mathrm{H} \cdots A$ \\
\hline $\mathrm{N} 9-\mathrm{H} 9 \cdots \mathrm{O} 8^{\mathrm{i}}$ & 0.86 & 2.08 & $2.935(3)$ & 179 \\
$\mathrm{C} 6-\mathrm{H} 6 A \cdots \mathrm{N} 10$ & 0.97 & 2.44 & $2.782(3)$ & 100 \\
$\mathrm{C} 13-\mathrm{H} 13 \cdots C g 1^{\mathrm{ii}}$ & 0.93 & 2.68 & $3.611(2)$ & 179 \\
\hline
\end{tabular}

Symmetry codes: (i) $-x,-y+2,-z+1$; (ii) $-x+1,-y+2,-z+1$. 